\title{
Teil IV: Im Pflegeheim
}

\author{
Katrin Bente Karl und Studierende
}

\section{Einleitung}

Katrin Bente Karl

In diesem Teil kommen größtenteils die studentischen Teilnehmerinnen zu Wort und teilen aus unterschiedlichen Perspektiven ihre Erfahrungen, die sie im Laufe ihrer Projektteilnahme sammeln. Da das Projekt nicht nur im universitären Kontext anzusiedeln ist, sondern einen umfangreichen praktischen Teil im Pflegeheim umfasst, soll an diesem Punkt zunächst die Perspektive der Pflegeheime eingenommen werden. Hier fügt eine Studentin die Ergebnisse von Interviews mit Kooperationspartnern zu einem Zusammenschnitt zusammen, den wir Polylog nennen und der Eindrücke davon verschafft, wie das Projekt, unsere Ansätze und die Umsetzung in den Einrichtungen wahrgenommen werden. Diesem Polylog schließt sich ein zweiter an, der ebenfalls die Ergebnisse von Interviews kondensiert: Hier werden die Erfahrungen von vier studentischen Projektteilnehmer/innen über die gesamte Projektlaufzeit gesammelt und in eine Chronologie gebracht. Die Besonderheit ist dabei, dass diese Teilnehmer/innen jeweils dieselbe Person über ein Jahr begleitet haben und sich nun rückblickend über sie und die Besuche bei ihr im Laufe der Jahre austauschen. Hier rückt eine konkrete begleitete Frau in den Mittelpunkt. Ziel dieses Abschnittes ist zu zeigen, wie kontinuierlich die Begleitung angelegt ist, welche Prozesse dabei beobachtet werden können und wie sehr der jeweils individuelle Mensch mit seinen Voraussetzungen und Möglichkeiten im Fokus des Projektes steht. Dieser Polylog soll stellvertretend für die

K. B. Karl und Studierende ( $\square)$

Seminar für Slavistik/Lotman-Institut, Ruhr-Universität Bochum, Bochum, Deutschland E-Mail: katrin.karl@rub.de 
vielen Paare stehen, die im Laufe des Projektes zueinandergefunden haben und in einen sehr privaten Austausch getreten sind. Dem schließen sich im umfangreichsten Abschnitt dieses Teils studentische Berichte an. Hier schildern jeweils einzelne Projektteilnehmerinnen mit unterschiedlichen Schwerpunkten ihre sehr persönlichen Eindrücke. Z. T. sind es Schilderungen von emotional schwierigen Situationen, die auch die traurigen und schweren Seiten der Projektarbeit thematisieren. In allen schwingt aber immer das tiefe Interesse an der begleiteten Person und die Verbundenheit zu ihr in all ihrer Unterschiedlichkeit mit. Diese Beiträge zeigen unmittelbar, welche Emotionen durch die Projektteilnahme entstanden sind und wie sehr sich die Studierenden mit der meist neuartigen Situation auseinandergesetzt haben und an ihr gewachsen sind.

\section{Die Stimme aus den Pflegeheimen: Das Projekt UnVergessen aus der Sicht der Kooperationspartner}

\section{Anna Danzeglocke}

Im folgenden Polylog wird es darum gehen, das Projekt UnVergessen aus der Perspektive der Kooperationspartner zu beschreiben, also aus der Sicht der Pflegeheime, die mit dem Projekt in Kontakt stehen. Dabei wird dargestellt, wie die Pflegeheime UnVergessen wahrnehmen, welchen Mehrgewinn das Projekt ihnen bringt, aber auch an welchen Stellen es noch Schwierigkeiten gibt. Außerdem wird darauf eingegangen, welche Auswirkungen das Projekt auf die Bewohner/innen der Pflegeheime hat und wie UnVergessen dabei helfen kann, eventuelle Probleme von Mehrsprachigkeit im Pflegeheim aufzugreifen. Für den Polylog wurden insgesamt drei Interviews mit Ansprechpartner/innen aus den Pflegeheimen geführt. Das erste Interview mit Herrn Ermers vom DRK Pflegezentrum Solferino in Essen wurde bereits im März 2019 von Yvonne Behrens durchgeführt. Im September 2020 folgten zwei weitere Interviews mit Frau Kersten vom Buchen-Hof in Bochum und Frau Stawinoga vom St. Marienstift in Bochum, welche von Anna Danzeglocke durchgeführt worden sind. Alle Interviewpartner/innen stehen mit dem Projekt UnVergessen schon mehrere Jahre in Kontakt, einige sogar schon von Anfang an. Aus diesem Grund konnten alle viel über UnVergessen und ihre Wahrnehmung des Projekts berichten. 
Interviewpartner/innen:

Dirk Ermers: $\quad$ Sozialarbeiter im Belegungsmanagement, DRK Pflegezentrum Solferino (Essen)

Petra Kersten: $\quad$ Hausleiterin, Buchen-Hof (Bochum)

Doris Stawinoga: Einrichtungsleiterin, St. Marienstift (Bochum)

Interviews durchgeführt von Yvonne Behrens und Anna Danzeglocke

Frau Kersten: $\quad$ Mit dem Projekt UnVergessen stehe ich schon, seitdem es begonnen hat, in Kontakt. Ich finde das Projekt wirklich genial!

Herr Ermers: $\quad$ Ich bin auch schon lange dabei und arbeite seit Ende 2017 mit dem Projekt zusammen.

Frau Stawinoga: Mit dem Projekt bin ich seit 2016 in Kontakt und bin von daher auch von Anfang an mit dabei gewesen. Das Projekt finde ich wirklich sehr gut. Es ist ein bisschen schade, dass wir im Moment nur eine russischsprachige Bewohnerin haben, die durch das Projekt begleitet wird.

Frau Kersten: Da kann ich mich nur anschließen. Ich finde das Projekt richtig gut und bin manchmal ein bisschen traurig, dass wir so wenige Bewohner haben, die neben Deutsch noch eine andere Sprache sprechen. Wenn wir viele unterschiedliche Sprachen hätten, dann könnte ich beim Projekt UnVergessen anrufen und sagen: „Ich bräuchte da mal jemanden für Kroatisch, Spanisch oder Italienisch“. Leider ist das im Moment nicht so. Wir haben derzeit keinen Bewohner, bei dem der Bedarf besteht, dass er von einem Studierenden besucht wird, da wir zum Beispiel nicht mit ihm kommunizieren können, weil er aus Polen oder Russland kommt.

Herr Ermers: $\quad$ Ich bin auch der Meinung, dass das Projekt UnVergessen ein riesiger Erfolg ist. Für mich ist das Besondere am Projekt, dass alle Beteiligten einen Gewinn haben, sowohl die Pflegeheime und die Bewohner als auch die Studierenden, die zu uns kommen. Ich habe auch bemerkt, dass die Bewohner, die von den Studierenden besucht werden, viel aktiver sind als vor der Teilnahme am Projekt, sie lassen sich leichter in die Gemeinschaft integrieren und sind auch fitter. Sie haben sogar Kontakt zu Bewohnern, mit denen sie vorher nicht so viel zu 


\begin{abstract}
tun hatten. Daran sieht man, dass das Projekt wirklich viele positive Auswirkungen auf die Bewohner hat.

Frau Stawinoga: Also bei der einen Bewohnerin, die momentan vom Projekt betreut wird, merkt man, dass sie sich jede Woche auf die Besuche freut und dass sie den Besuch auch schon kennt. Für die Bewohnerin sind die wöchentlichen Besuche immer wieder ein Highlight. Deswegen kann ich Ihnen nur zustimmen, Herr Ermers, dass das Projekt sich positiv auf die Bewohner auswirkt. Es ist für uns auch schon normal geworden, dass ein Studierender zu uns kommt und einen Bewohner begleitet.
\end{abstract}

Frau Kersten: $\quad$ Im Moment haben wir zwar keinen Bewohner, der durch einen Studierenden begleitet wird, aber bei denen, die teilgenommen haben, hatte das Projekt die Auswirkung, dass sowohl die Studierenden als auch die Bewohner sich aufeinander gefreut haben und begeistert voneinander waren. Das hat einen Einfluss darauf, dass es den Bewohnern gut geht. Es trägt dazu bei, die Bewohner ausgeglichener zu machen, fröhlicher und ein bisschen ruhiger, weil da jemand ist, der sich für eine Stunde nur für diesen einen Bewohner Zeit genommen hat. In anderen Worten wird dem Bewohner so gezeigt: „Du bist mir wichtig, und ich komme auch nur für dich“. Und das hat positive Auswirkungen. Deshalb sehe ich in dem Projekt auch eigentlich keine Schwierigkeiten. Nur bei der Kommunikation hapert es manchmal ein bisschen.

Herr Ermers: $\quad$ Schwierigkeiten haben wir mit dem Projekt eigentlich auch keine. Das ist meiner Meinung nach auch immer ein bisschen Erfahrungswert. Manchmal gibt es zum Beispiel Probleme bei der Verbindlichkeit, dem regelmäßigen Kontakt oder den regelmäßigen Besuchen. Insbesondere für demenzerkrankte Bewohner ist wichtig, dass eine Kontinuität herrscht.

Frau Stawinoga: Ich sehe das ähnlich. Eigentlich gibt es mit dem Projekt UnVergessen keine Schwierigkeiten, nur manchmal etwas in der Kommunikation. Da müssen aber meiner Meinung nach auch beide Seiten dran arbeiten, also das Projekt genauso wie die Kooperationspartner. Schwierig ist natürlich auch immer die Situation, wenn der Studierende, der unsere Bewohnerin betreut, wechselt. Das ist dann jedes Mal ein Neuanfang, das kann man sich ja vorstellen. Die Bewohnerin muss erst wieder Vertrauen zu dem Studierenden finden, und es dauert dann 
Frau Kersten: Das sehe ich genauso. Natürlich hätten wir gerne vier Jahre hintereinander jede Woche den gleichen Studierenden, der einen Bewohner besucht. Aber das ist unrealistisch, und wir wissen das auch. Wir haben immer versucht, das so hinzubekommen, dass die Bewohner sich darauf einlassen. Das hat bei einer Bewohnerin auch sehr gut funktioniert. Sie wurde über die Semester von unterschiedlichen Studierenden betreut.

Herr Ermers: Ja, da kann ich mich nur anschließen. Es ist natürlich schwierig, eine Kontinuität hinzukriegen, die ja gerade für die demenzerkrankten Bewohner sehr wichtig ist. Aber da stößt man ein bisschen an seine Grenzen. Man muss versuchen, den Übergang zu gestalten, und dann im Einzelfall gucken, wie die Bewohner damit zurechtkommen. Das kann funktionieren, aber pauschal einschätzen kann man das nicht. Entweder der Übergang gelingt oder eben nicht. In jedem Fall haben wir durch UnVergessen ein weiteres Angebot. Das ist ein Mehrwert, den wir anbieten können, weil wir schlecht in Abhängigkeit von den Sprachen der Bewohner das Pflegepersonal einstellen können. Und wenn es einen Studierenden gibt, der einen Bewohner begleitet, dann ist das einfach ein großer Gewinn. Ansonsten gibt es bei uns bisher auch keine konkreten Ansätze, auf unterschiedliche Sprachen und Kulturen einzugehen, einfach weil der Bedarf mit 10-15\% mehrsprachigen Bewohnern nicht besteht.

Frau Stawinoga: Bei uns können vor allem die Mitarbeiter gut auf unterschiedliche Sprachen eingehen, da wir Personal aus vielen verschiedenen Herkunftsländern haben. So haben wir zum Beispiel auch eine russischsprachige Pflegerin, die sich mit unserer einen Bewohnerin auch auf Russisch unterhält. Durch das Projekt UnVergessen kommen aber auch immer neue Aspekte in unser Pflegeheim, und wir öffnen uns ein Stück weit nach außen. Man sollte immer offen sein für neue Dinge. Von Projekten wie UnVergessen kann man eigentlich nur profitieren. Man bekommt neue Ideen und sieht auch, an welchen Stellen man neue Aspekte in die Pflege einbringen kann. 
Frau Kersten: Besonders dieses Öffnen nach außen ist für uns auch eine gute Gelegenheit, um zu zeigen, wie stationäre Altenpflege aussieht. Wenn die Studierenden kommen und sehen, wie wir uns kümmern und wie wir arbeiten, können wir vielleicht zu einem ganz kleinen Teil dazu beitragen, dass sich die Ansicht von stationärer Pflege dadurch ein bisschen verändert. Außerdem ist UnVergessen eine tolle Möglichkeit für die Menschen, die zu uns ziehen, ihre Sprache weiterzupflegen. Wir werden auch immer wieder mal Bewohner haben, die mehrsprachig sind. Unser Problem ist dann, wenn wir die Bewohner mit der deutschen Sprache nicht mehr erreichen. Dann ist es super, wenn man UnVergessen um Hilfe bitten kann.

Frau Stawinoga: Das sehe ich auch so. Ich erinnere mich auch noch an eine Situation, bevor wir mit dem Projekt UnVergessen zusammengearbeitet haben, wo wir eine Bewohnerin hatten, die mehrsprachig war. Allerdings konnte sie das Deutsche nicht mehr aktiv nutzen, und wir hatten keinen Mitarbeiter, der ihre Muttersprache konnte. Da hatten wir bei der Kommunikation massive Probleme. An der Stelle hätte ich mir schon jemanden gewünscht, der uns dabei unterstützt. Und eine ähnliche Situation kann ja erneut vorkommen. Jetzt haben wir die Möglichkeit, uns dann an UnVergessen zu wenden. Außerdem haben wir bei uns auch einmal eine Präsentation von UnVergessen gehabt, in der vorgestellt wurde, wie man mit nicht deutschsprachigen Bewohnern kommunizieren kann, die demenzerkrankt sind. Das war sehr wertvoll für uns.

Herr Ermers: $\quad$ Darin sehe ich auch eine gute Perspektive für UnVergessen. Vor allem die Vermittlung einfacher Sprachkenntnisse wäre sehr hilfreich. Wenn man keine Mitarbeiter hat, die dieselbe Sprache sprechen wie der Bewohner, kann das bei der Verständigung helfen. Insgesamt kann ich damit sagen, dass ich die Teilnahme an UnVergessen nur weiterempfehlen kann.

Frau Stawinoga: Da kann ich mich nur anschließen.

Frau Kersten: Ja, ich auch!

An dieser Stelle erneut vielen Dank an unsere Interviewpartner/innen für ihre Zeit und die interessanten Gespräche! 


\title{
Die Stimme der Bewohner/innen: Porträt einer russischsprachigen Pflegebedürftigen aus der Perspektive „ihrer" Studierenden
}

\author{
Julia Golbek
}

Frau Schneider ${ }^{1}$ ist eine Frau, die seit mehreren Jahren in einem Pflegeheim lebt. Sie ist in Russland geboren, dort einsprachig Russisch aufgewachsen und kam im Laufe ihres Berufslebens nach Deutschland. Wann und wie lange sie in deutschsprachiger Umgebung gelebt und vielleicht auch gearbeitet hat, ist unbekannt. Ebenfalls fehlt konkretes Wissen über ihre Deutschkenntnisse. Frau Schneider hat keine direkten Verwandten, die sie besuchen. Sie hat eine vor mehreren Jahren diagnostizierte Demenz und wies bereits bei ihrem Einzug ins Pflegeheim Erinnerungslücken auf. Zudem zählt sie nicht zu den Menschen, die von sich aus viel über sich berichten. Erschwerend kommt hinzu, dass sie mit dem Pflegepersonal ihrer Einrichtung überwiegend nonverbal kommuniziert, was vielfach auf die Sprachbarriere zurückgeführt wird. Frau Schneider spricht kaum Deutsch, scheint es aber gut zu verstehen und reagiert zugewandt und freundlich mit allen ihr zur Verfügung stehenden körperlichen Ausdrucksmöglichkeiten. In der Folge ist vieles über Frau Schneiders Leben, ihre sprachlichen Kenntnisse, kommunikativen Möglichkeiten und den Status ihrer demenziellen Erkrankung unklar.

In der ersten Runde des Projektes UnVergessen im Jahr 2017 wurde Frau Schneider von Johann Funk besucht. Mit dem Kennenlernen der Beiden begann das Projekt, weswegen Frau Schneider als Teilnehmerin der ersten Stunde angesehen werden kann. Im Folgenden stellte Johann Funk sie, die Gespräche und gemeinsam verbrachte Zeit über die Dauer des ersten Jahres in den Gruppentreffen dar. Georg Stin nahm in der zweiten Runde am Projekt UnVergessen teil und „übernahm“ die Begleitung von Frau Schneider. Er besuchte sie von Januar bis Juli 2018 und sammelte seinerseits neue Erfahrungen in den gemeinsamen Treffen. Diese Erfahrungen teilte er mit Erika Erhardt, die im dritten Projektdurchlauf zwischen Februar und Juli 2019 die Besuche bei Frau Schneider fortsetzte. Auch sie baute ein eigenes und wiederum individuelles Verhältnis zu ihr auf. In der aktuellen Runde wird Frau Schneider seit Januar 2020 von Julia Golbek begleitet, wobei der Kontakt aufgrund des Besuchsverbots wegen des Coronavirus seit Mitte März über das Telefon stattfindet. In diesem Polylog berichten die Personen, die im Laufe der letzten Jahre Frau Schneider kennengelernt und ein Stück

${ }^{1}$ Der Name wurde geändert. 
ihres Weges begleitet haben, von den Erfahrungen mit ihr, der gemeinsam verbrachten Zeit, den Herausforderungen und auch den Veränderungen, die sich in der Zusammenschau im Laufe ihrer Projektteilnahme beobachten lassen.

\section{Gesprächsteilnehmer/innen:Georg Stin, Erika Erhardt und Julia Golbek}

Georg: Als ich Frau Schneider besucht habe, war es für mich unmöglich, einen Alltagsdialog mit ihr aufzubauen. Es kam mir so vor, als wäre es für sie anstrengend und schwer, ein „trockenes“ Gespräch zu führen. Deshalb habe ich immer die Initiative ergriffen und die Gespräche angefangen. Außerdem habe ich ihr Bildbände mitgebracht, um das Gespräch aufrechtzuerhalten. Ich dachte, Bildbände wären eine gute Idee, da sie das Papierformat kennt. Die Fotos darin sind ja auch meist etwas älter. Deshalb nahm ich an, Frau Schneider könne diese gut mit ihren Erinnerungen verknüpfen.

Erika: Frau Schneider und ich haben uns auch meist Bilder angeschaut. Ich hatte sie sowohl auf Papier als auch auf meinem Laptop dabei. Außerdem hat Frau Schneider mir gesagt, wo sie herkommt, und ich habe ihr Fotos von ihrer Heimatstadt mitgebracht.

Julia: $\quad$ Ich habe Frau Schneider auch oft Bilder mitgebracht, allerdings eher in Form eines Sprachstandstests ${ }^{2}$ für bilinguale Kinder. Sie hat sich immer über diesen Test gefreut. Nach dem Test habe ich sie ab und zu gebeten, alle Bilder zu benennen, was sie ohne große Probleme gemacht hat. Außerdem haben wir uns über die gemalten und gar gestickten Bilder im Zimmer von Frau Schneider unterhalten. Hier habe ich das genauso gemacht wie du, Georg, ich habe sie dann von dem Bild ausgehend gefragt, ob sie zum Beispiel schon einmal am Meer war.

Georg: Ja, ich habe sie genauso zum Sprechen animiert. Wenn wir zum Beispiel ein Foto von einem Markt angeschaut haben, haben wir über Essen und Obst gesprochen. Ich hatte ab und zu einen Bildband „Moskau“ dabei, woraus Frau Schneider sehr gerne kommunistische Losungen vorgelesen hat.

Erika: Ich habe für sie auch jede Woche Aufgaben vorbereitet. Frau Schneider hat sie immer gelöst und hat sehr gut darauf reagiert, weil sie dabei auch gefordert wurde.

Julia: Ach, mir ist etwas Interessantes eingefallen. Als ich Frau Schneider fragte, ob sie gerne singe, hat sie ein russisches Lied über den Tannenbaum angestimmt. Im Laufe des Projekts habe ich festgestellt, dass

\footnotetext{
${ }^{2}$ Hier ist der Sprachstandstest von Gagarina et al. (2010) gemeint.
} 
sie sich an mehrere Lieder erinnern kann. Eins davon hatte sogar einen veränderten, lustigen Text im Vergleich zum Original. Da Frau Schneider am Singen wirklich Spaß hat, habe ich mit ihr zusammen gesungen, obwohl meine Stimme nicht unbedingt die beste ist.

Erika: Ich habe auch schon festgestellt, dass Frau Schneider Musik mag und mitsingt, wenn man ein Lied anstimmt. Das habe ich beobachtet, als eine Pflegekraft im Gemeinschaftsraum ein russisches Lied anstimmte und Frau Schneider anfing, laut mitzusingen. Ich selbst habe das mit ihr aber nicht ausprobiert. Schön, dass es bei euch geklappt hat!

Julia: Da Frau Schneider in meiner Runde wahrscheinlich noch weniger gesprochen hat als bei euch, habe ich sie oft gefragt, worüber ich ihr denn etwas erzählen soll. Meist waren es persönliche Themen oder etwas Allgemeines, wie die Taiga. Hierbei habe ich immer versucht, sie in das Gespräch miteinzubeziehen, indem ich zum Beispiel über meine Familie erzählte und sie im Anschluss nach ihrer fragte.

Georg: Das war für mich auch nicht leicht, sie zum Sprechen zu bewegen. Es ist ja sinnlos, direkte Fragen wie „Was sind Ihre Interessen?“ zu stellen, weil Frau Schneider dann mit „keine“ oder ,nichts“ antwortet. Ich habe auch festgestellt, dass sie beim Thema Familie oder Kindheit eher dazu bereit war, ins Gespräch zu kommen und etwas über sich zu erzählen. Als ich das erste Mal bei ihr war, hat sie fast alle meiner Fragen ignoriert, bis auf die, in denen es um ihre Familie ging. Als sie diese beantwortet hat, hat sie sogar gelächelt.

Erika: Bei mir war das so ähnlich wie bei euch. Frau Schneider sprach nie aus eigener Initiative und antwortete auf meine Fragen nur mit 1-2 Wörtern. Oft hat sie aber nur genickt, manchmal sogar lächelnd, wenn ich ihr etwas erzählt und dann gefragt habe, ob sie das interessiert und ich weitererzählen soll. Und da sie sich nicht an mich erinnern konnte, habe ich ihr öfter über meine Familie erzählt. Von Johann, der Frau Schneider in der ersten Runde betreut hat, habe ich gehört, dass sie sich an seinen Namen erinnern konnte. Und das trotz Demenz im frühen Stadium!

Julia: Ja, genau, so war das bei mir auch. Sie konnte sich nicht an mich erinnern, aber sie war manchmal in der Lage, sich an einige Aussagen von mir während des ganzen Besuchs zu erinnern. So habe ich sie am Ende eines Besuchs gefragt, was ich das nächste Mal mitbringen soll und sie sagte „Fotos von der Schwester“. Ich war wirklich beeindruckt, weil wir uns vielleicht eine halbe Stunde vorher über unsere Geschwister ausgetauscht hatten. Sie spricht grundsätzlich gerne über Familie. Ich 
erinnere mich daran, dass Johann berichtet hat, dass sie mit ihm über ihren Mann und ihre Tochter, aber auch über Politik sprach.

Georg: Ich denke, dass alles, was mit ihrer Familie verbunden ist, bei Frau Schneider doch noch präsent ist. Es ist bemerkenswert, an welche Kleinigkeiten sie sich erinnern kann, wenn es um ihre Familie oder Kindheit geht, aber an meine wöchentlichen Besuche nicht. Sie hat mir den Weg zur Datscha beschrieben, über die Ausstattung der Räume berichtet und davon erzählt, wie ihre Mutter die Schlüssel verloren hat und wo sie gehangen haben. Das unterscheidet sich meiner Meinung nach nicht von Erinnerungen einer nicht demenziell erkrankten Person. Beim Smalltalk fiel der Unterschied aber umso mehr auf. Sie spricht ja selten und man muss ihr signalisieren, dass man eine mündliche Antwort statt zum Beispiel ein Kopfnicken erwartet. In der ganzen Zeit, die ich sie besucht habe, hat sie mir nur zwei einfache Rückfragen, „und du?“, gestellt. War das bei euch auch so?

Erika: Mit mir hat Frau Schneider auch eher nonverbal kommuniziert. Für Themenwechsel hat sie selbst keine Vorschläge gemacht, aber sehr wohl geantwortet oder eben genickt, wenn ich sie gefragt habe, ob wir uns über dies oder jenes unterhalten wollen.

Julia: Ich habe das auch so erlebt. Allerdings habe ich versucht, ihr für die Antwort etwas Zeit zu geben oder habe zwischen meinen Sätzen Pausen gemacht. Diese Pausen hat sie manchmal selbst gefüllt. Momentan halte ich mit Frau Schneider telefonisch Kontakt und habe festgestellt, dass sie am Telefon mehr sagt. In der Regel meldet sie sich am Telefon mit einem ,Hallo‘, um zu signalisieren, dass sie jetzt dran ist. Das ist sehr russisch! Insgesamt habe ich das Gefühl, dass ihr das Telefonieren zum Teil sogar leichter fällt und von ihr auch mehr kommt. Ich denke, dass ihr bewusst ist, dass sie eine mündliche Antwort geben muss, und deswegen hört man von ihr auch „Ja“ oder „Nein“. Man muss sie aber genauso wie bei Besuchen ausfragen und eine Frage auch wiederholen, wenn gerade keine Antwort kommt. Wie sah denn eine typische Situation aus, die ihr während eines Besuchs erlebt habt?

Erika: Ein typischer Besuch begann damit, dass ich sie auf Russisch fragte, wie es ihr geht. An ihrer Antwort konnte ich bereits einschätzen, ob sie an diesem Tag gut drauf war. An guten Tagen antwortete sie mit einem klaren ,gut“ und an weniger guten Tagen reagierte sie gar nicht auf die Frage oder lächelte nur. Zu Beginn führte ich ein wenig Smalltalk mit ihr. Manchmal stellte ich mich neu vor, manchmal auch nicht. Meistens hatte ich ein Spiel oder eine Aufgabe dabei, die wir als erstes lösten, 
solange ihre Aufmerksamkeit noch da war. Danach schauten wir uns gemeinsam Bilder an, und ich erzählte etwas zu den Fotos und stellte ihr nebenbei Fragen, um etwas mehr von ihr zu erfahren. Wenn sie etwas interessiert hat, dann hat sie gelächelt und hatte beide Augen geöffnet. Bei Desinteresse hatte sie oft nur ein Auge geöffnet.

Julia: Genauso habe ich das auch erlebt! Da sie im Rollstuhl sitzt, hat sie sich manchmal von mir wegbewegt, indem sie sich mit den Füßen vom Boden abgestoßen hat. Sie kann also ihrem Gegenüber mitteilen, wenn das Thema sie nicht interessiert, nur eben auf ihre eigene Art. Ab und zu hat sie ihren Kopf auf den Tisch gelegt. Hier weiß ich aber nicht genau, ob sie nicht eher müde war. Ich habe sie dann immer gefragt, ob sie müde sei und ob ich jetzt gehen und sie ausruhen lassen solle. Sie gab mir aber zu verstehen, dass sie zwar müde sei, ich aber noch bleiben könne.

Georg: Die nonverbale Kommunikation ist für Frau Schneider, wie auch für alle demenziell Erkrankten, sehr typisch. Sie antwortet ja oft mit einem Kopfschütteln oder Nicken auf eine Frage. Aber sie teilt sich auch durchs Lachen oder Lächeln mit, wenn ihr etwas gefällt. Johann sagte zu mir, dass man es Frau Schneider förmlich ansähe, dass sie aktiver geworden sei und es genieße, Russisch zu sprechen. Laut ihm reichte mit der Zeit schon ein Wort, um sie zum Sprechen zu animieren. Also kann man festhalten, dass die kommunikativen Fähigkeiten von Frau Schneider im ersten Projektjahr noch deutlich besser waren. Das ist der Beweis dafür, dass Menschen von UnVergessen profitieren. Die Kommunikation mit den deutschsprachigen Pflegekräften hingegen findet, so denke ich, eher einseitig statt.

Julia: Bei dem letzten Punkt bin ich mir nicht so sicher. Ich habe miterlebt, wie eine Pflegerin Frau Schneider wiederholt aufgefordert hat, mit ihr auf Deutsch zu sprechen. Ich wusste bereits, dass Frau Schneider relativ gut Deutsch versteht und habe abgewartet, was sich aus der Situation ergibt. Die Pflegerin hat Frau Schneider zum Beispiel mehrmals gefragt, wen wir in ihrem Zimmer antreffen werden, wenn wir gleich durch die Tür kommen. Frau Schneider hat dann nach dem zweiten oder dritten Mal tatsächlich auf Deutsch geantwortet und sagte sehr deutlich ,meinen Bruder". Wie wir wissen, hat sie keinen Bruder, aber sie hat sich sehr wohl auf Deutsch mitteilen können. Und das ist in Anbetracht ihres Demenzstadiums unglaublich!

Georg: Das ist wirklich interessant! Ich habe nur mitbekommen, dass Frau Schneider den Pflegekräften wortlos antwortet. 
Erika: Regulär haben Frau Schneider und ich auch nur Russisch gesprochen. Aber bei manchen Aufgaben habe ich sie bewusst danach gefragt, wie ein bestimmtes Wort auf Deutsch heißt und sie kannte die deutsche Bezeichnung. Bei diesen Begriffen handelte es sich um Lebensmittel oder Gegenstände aus ihrem Alltag.

Georg: Ja, genau, die alltägliche deutsche Lexik kennt sie. Meistens nennt sie die Wörter aber nicht spontan, sondern benutzt zuerst die russische Entsprechung.

Julia: $\quad$ Ähnliches habe ich auch bei dem Sprachstandstest beobachtet. An einer Stelle sollte Frau Schneider die ihr gezeigten Bilder auf Deutsch benennen. Ich habe sie in dieser Situation stets auf Deutsch angesprochen, aber sie hat zuerst auf Russisch reagiert. Danach habe ich sie nach dem deutschen Pendant gefragt, und sie konnte fast immer das richtige nennen.

Erika: Die Assoziationsaufgaben hat Frau Schneider mit mir auch sehr gut gelöst und mehr Begriffe genannt, als ich anfangs gedacht hatte.

Julia: Etwas Vergleichbares habe ich mit Frau Schneider auch gemacht. Ich habe mich hingestellt und sie gebeten, die Körperteile, auf die ich zeigte, auf Russisch zu benennen. Bei dieser Aufgabe hatte sie natürlich keine Probleme. Im Anschluss habe ich gefragt, ob wir das auf Deutsch ausprobieren wollten und zeigte auf dieselben Körperteile noch einmal. Sie konnte mir auch auf Deutsch alles benennen.

Georg: Ich habe Frau Schneider übrigens einmal danach gefragt, ob sie Deutsch spreche. Sie antwortete „Ja, aber nur ein bisschen“. Dieses Gespräch führten wir natürlich auf Russisch. Ich habe aber in Erfahrung bringen können, dass sie in Deutschland keine Ausbildung abgeschlossen und zu Hause nur Russisch gesprochen hat. Sie hat auch nicht lange in Deutschland gearbeitet. Von daher bin ich davon ausgegangen, dass ihr Deutsch früher nicht perfekt war. Sie kann aber auf Deutsch lesen und sogar vorlesen. Von daher denke ich, dass das, was man in seinem Leben gelernt oder erlebt hat, zu einem gewissen Grad auch bei demenziell Erkrankten abrufbar ist. Man kann vor allem festhalten, dass man am Beispiel von Frau Schneider ganz gut sieht, dass trotz Demenz viele Erinnerungen bleiben. Man muss da vielleicht nur etwas nachhelfen und gezielt Fragen stellen. Man ist immer eine Frage von einer interessanten Geschichte entfernt. 


\section{Die Stimme der Studierenden:Wie nehmen die Studierenden ihre Projektarbeit wahr?}

\section{Einleitung}

Katrin Bente Karl

In diesem Abschnitt werden von insgesamt sechs studentischen Teilnehmerinnen Erfahrungsberichte wiedergegeben. Diese reflektieren die Teilnahme am Projekt und arbeiten unterschiedliche Facetten der Erfahrungen auf. Dabei konzentrieren sich die Berichte jeweils auf sehr individuelle Punkte und greifen wichtige Elemente der jeweiligen Beziehung zwischen Studentin und Pflegebedürftigem auf. Die Berichte sind dabei bewusst vielfältig und sollen damit als Spiegel für die Heterogenität und unterschiedlichen Konstellationen stehen. Aus ihnen wird ersichtlich, wie unterschiedlich die Gesprächsthemen, Aktivitäten und auch die verwendeten Sprachen sind. Auch darin liegt eine der Besonderheiten der hier aufgebauten Beziehungen: Im Fokus steht der jeweilige Mensch mit seinen Bedürfnissen und Möglichkeiten, und darauf ist die jeweilige Besuchskonstellation sehr fein abgestimmt. So ist auch zu begründen, weswegen nicht in allen Fällen die Sprache der Kommunikation durchgehend Russisch oder Polnisch ist. Vielfach wird - wie auch in anderen zweisprachigen Settings - die Sprache gewechselt, dem Thema oder auch dem Kommunikationsumfeld angepasst. Sei es, dass bestimmte Wörter in der einen Sprache vertrauter sind oder eine andere Person den Raum betritt. Neben solchen zweisprachigen Gesprächen treten auch einsprachige auf. So ist z. B. eine der begleiteten Frauen so weit in ihrer Demenz fortgeschritten, dass sie nur mehr ausschließlich auf Russisch reagiert und sich selbst äußert. Eine Ansprache auf Deutsch führt zu unkontrollierbaren Ausbrüchen. Während auf der anderen Seite eine andere Teilnehmerin als Kind von Polen in Deutschland geboren wurde und während ihrer Kindheit aufgrund ihrer Herkunft diskriminiert wurde. Im Laufe ihres Lebens hat sie sich mit Fragen der Identität und Zugehörigkeit auseinandergesetzt. Für sie hat das Polnische einen sehr besonderen Stellenwert, da es sie einerseits an ihre Kindheit erinnert, andererseits aber auch viele negative Konnotationen trägt. Grundsätzlich fühlt sie sich in der deutschen Sprache sicherer und ihr verbunden. Sie kann durchaus stellvertretend für ein interessantes Phänomen angesehen werden, auf das wir im Laufe des Projektes bereits mehrfach gestoßen sind: Sie empfindet sich selbst als Deutsche, dabei wird sie jedoch von Anderen als Polin angesehen. Auf der Grundlage dieser Wahrnehmung ist sie auch als Teilnehmerin von dem Pflegeheim vorgeschlagen worden, obwohl sie als ihre Muttersprache Deutsch ansieht und sich 
kaum traut, Polnisch zu sprechen. Nicht zuletzt auch, weil sie über eine vermeintlich verkehrte Sprachwahl Nachteile für sich befürchtet. Besonders schön erscheint in diesem Fall, dass diese Frau sich emotional der Studentin geöffnet hat, die sie besuchen kam. Milena Ploch hat immer wieder polnische Sätze in die sonst überwiegend deutschen Gespräche eingebaut. Frau Spiller hat Milena liebevoll ,ihre kleine Polin“ genannt und sich über und auf ihre Besuche gefreut und sich zunehmend getraut, auch eigene Sätze, Lieder und Gedichte auf Polnisch einzustreuen. Dies zeigt, welches Vertrauen hier aufgebaut und vielleicht sogar Ängste abgebaut werden konnten.

Aus allen der folgenden Berichte spricht eine ähnliche tiefe Erfahrung und Ehrfurcht davor, dass sich ein zuvor unbekannter Mensch im Laufe der Zeit unabhängig von Altersunterschieden, Krankheiten und z. T. auch traumatischen Erfahrungen - öffnet und den Anderen an seinen Gedanken und Emotionen teilhaben lässt. Dies führte zu vielen schönen Momenten, zugleich aber auch zu manchen traurigen und schweren, da eben auch die Kriegserinnerungen oder Altersängste geteilt wurden.

Was in der Rückschau aus diesen so individuellen und besonderen Beziehungen bei den Studierenden geworden ist, zeigen nun diese Berichte hier. Diese bestehen größtenteils aus den eigenen Erinnerungen, z. T. finden sich dabei Verweise auf wissenschaftliche Literatur und entsprechende Diskurse. Vorrangig der letzte Beitrag bettet sich in einen wissenschaftlichen Kontext ein und kann damit bereits als Übergang zum Folgekapitel aufgefasst werden, in dem die wissenschaftliche Perspektive wiederum eine größere Rolle spielt.

Den Auftakt bildet die Darstellung eines gemeinsam verbrachten Nachmittags zwischen Sabina Safarova und ihrer russischsprachigen Pflegebedürftigen, die stellvertretend für die sehr besondere Beziehung zwischen den Beiden steht. Wie dort zwischen den Zeilen anklingt, zeigt die an Demenz im Endstadium erkrankte Pflegebedürftige ein herausforderndes Verhalten, das nicht selten auch erfahrene Pflegekräfte belastet. Sabina Safarova gelang es, dennoch eine Beziehung zu ihr aufzubauen und ihren Humor und Mut nicht bloß zu entdecken, sondern aufzugreifen und zu fördern. Schon kurze Zeit nach ihrem Kennenlernen war klar, dass sich hier zwei Seelen gefunden haben, die eine gemeinsame Sprache sprechen und auf diese Weise viele sehr besondere Stunden miteinander teilen konnten.

Auch aus dem zweiten Bericht - von Milena Ploch, die eine in Polen geborene Frau besuchte - spricht eine herzliche Wärme. Er zeigt, wie viel Freude und schöne Momente miteinander geteilt wurden. Hier steht die schöne Botschaft, dass ein Lächeln ein wertvolles Geschenk und überdauernde Erinnerung ist und die Brücke zwischen den Generationen schlagen kann. 
In der dritten Rückschau auf die Projektteilnahme berichtet Lydia Brodovski von ihrer ursprünglichen Idee, zwei Methoden in den Gesprächen mit ihrer Person zu kombinieren und so der Frage nach dem Verständnis von Heimat in der besonderen biografischen Konstellation einer mehrfach migrierten und in wechselnden Lebensverhältnissen wohnenden Person nachzugehen. Auf welche Probleme und anschließende Überlegungen sie dabei stieß, ist dort einfühlsam zu lesen.

Der vierte Beitrag widmet sich einer ganz besonderen Beziehung zwischen Yvonne Behrens und einem polnischsprachigen Mann, den sie über mehrere Jahre hinweg regelmäßig besucht hat. Yvonne Behrens hat an der ersten Runde des Projektes teilgenommen und ist so begeistert in das Projekt eingestiegen, dass sie auch nach ihrer studentischen Teilnahme dabeiblieb. Es entwickelte sich eine Mitarbeit im Projekt als wissenschaftliche Mitarbeiterin, ein tiefes wissenschaftliches Interesse an dem Themenkomplex Demenz und Mehrsprachigkeit und nicht zuletzt eine ganz besondere Bindung zu ihrer Besuchsperson. Wie diese Beziehung entstand und sich über die Jahre entwickelte, veränderte und vertiefte, schildert sie in ihrem Bericht.

Dem schließt sich eine Darstellung von Dorothea Laszczak und ihren ebenfalls sehr emotionalen Erfahrungen an. Sehr eindrücklich schildert sie u.a., wie sie mit der Nachricht des Todes einer der zwei von ihr besuchten Frauen umgehen musste. Dies ist eine sehr traurige Angelegenheit, die aber leider durch die Pflegeheimsituation nicht unwahrscheinlich ist und daher häufig im Hintergrund mitschwingt. Die Trauer auf diese Nachricht zeigt auch hier, welche Bindung durch die regelmäßigen Besuche entstanden ist.

Der letzte Beitrag in diesem Kapitel ist von Aldona Rzitki geschrieben, die sich der Frage widmet, wie man im Gespräch mit einer dementen Person passend reagiert, wenn man der Überzeugung ist, dass das von ihr Geschilderte nicht den üblichen Kriterien der Wahrheit entspricht. Auch aus dieser Darstellung wird deutlich, dass sich Aldona Rzitki tiefgehende Gedanken über den bestmöglichen Umgang mit ihrer besuchten Person macht und sich der Frage stellt, welchen Sinn die Besuche und damit die gemeinsam verbrachte Zeit verfolgen. Dieser Bericht ist unterfüttert mit Verweisen auf wissenschaftliche Literatur und stellt damit ein Bindeglied zum darauffolgenden Kapitel mit seiner wissenschaftlicheren Fokussierung dar. 


\section{Ein unvergesslicher Frühlingstag}

\section{Sabina Safarova}

Edeltraud $^{3}$ war eine außergewöhnliche Dame. Wir kannten uns nun schon seit einigen Monaten. Doch heute sollte ein ganz besonderer Tag für uns beide werden. Es war ein wunderschöner Frühlingsmorgen, zumindest für mich. Edeltraud schien jedoch wenig begeistert, als ich ihr Zimmer betrat und ihr vorschlug, einen Spaziergang zu machen. So machten wir es doch üblicherweise, wenn ich sie besuchte. $\mathrm{Ob}$ sie sich noch daran erinnerte, fragte ich mich, während ich sie für den Spaziergang vorbereitete. Ob sie sich überhaupt an mich erinnerte?

Über Edeltraud wusste ich, dass sie ein stolzes Alter von 95 Jahren erreicht hat und in Russland geboren war. Sie war verheiratet und hat zwei Töchter. Den Erzählungen ihrer Töchter und der Pflegekräfte zufolge, auch belegt durch die vielen liebevoll dekorierten Fotos in ihrem Zimmer, war Edeltraud in ihrem ehemaligen Beruf Ärztin und Direktorin einer Poliklinik in Russland.

Die Sonne schien, die Vögel zwitscherten und die Bauarbeiten vor der Pflegeeinrichtung traten für eine kurze Zeit in den Hintergrund. Obwohl Edeltraud zunächst wenig motiviert und begeistert schien, konnte ich sie doch noch davon überzeugen, gemeinsam mit mir an die frische Luft zu gehen. Wir nutzen die Gelegenheit und machten uns auf, spazieren zu gehen...

„Schneller, schneller, schneller“, schrie Edeltraud auf Russisch, als wir mit dem Rollstuhl die Straße überquerten. Edeltraud konnte es nie schnell genug gehen. Ich versuchte etwas schneller zu laufen und schob den Rollstuhl, so schnell es mir möglich war, vorwärts vor mir her.

„Schneller, schneller, schneller!“, rief Edeltraud erneut. Aber schneller war es mir nun mal mit dem Rollstuhl nicht möglich. Es war schließlich nur ein Rollstuhl und kein Quad. Zumal ich in meiner Betreuungszeit die Aufgabe hatte, für ihre Sicherheit zu sorgen. Hinzu trat auch noch, dass ich sehr unerfahren im Umgang mit dem Rollstuhl war und keine Fehler machen wollte, die Edeltraud oder mich in Schwierigkeiten hätten bringen können.

Seit dem ersten Augenblick, als ich Edeltraud begegnet war, war ich von ihrer Risikofreudigkeit, ihrer Direktheit und ihrem Mut tief beeindruckt. Sie war die mutigste, draufgängerischste und unverschämteste, aber auch liebenswürdigste Seniorin, die ich je kennengelernt hatte. Es schien, als habe sie vor nichts und niemandem Angst. Edeltraud nahm kein Blatt vor den Mund. Wenn ihr etwas nicht passte, kriegte man es deutlich zu spüren.

\footnotetext{
${ }^{3}$ Der Name wurde geändert.
} 
„Dura, Dura, Dura!“”, schrie Edeltraud nun lauthals und mit aller Kraft auf Russisch, was übersetzt ins Deutsche unter anderem Idiotin oder blöde Kuh bedeutete. So nannte sie mich öfter, wenn sie unzufrieden war oder sich gestresst fühlte. Gelegentlich schmiss sie auch mit Gegenständen um sich. Ich nahm es ihr nicht übel. Denn ich verstand, dass ihre Situation keine einfache war.

Aus ihren sprachbiografischen Angaben und aus den Gesprächen mit ihren Pflegekräften und ihren beiden Töchtern wusste ich bereits, dass Edeltraud durch ihre sehr fortgeschrittene Demenz die deutsche Sprache inzwischen fast vollständig vergessen hatte. Sie sprach nur noch einige vereinzelte Wörter und Sätze, und diese auch ausschließlich in der russischen Sprache, also ihrer Kindheitssprache.

Dies führte zwangsläufig zu vielen Kommunikationsschwierigkeiten in ihrem Alltag mit Angehörigen und Pfleger/innen und stellte sie vor große Herausforderungen. Doch am schwierigsten war es wohl für Edeltraud selbst.

Wir gingen weiter spazieren und ich versuchte Edeltraud dazu zu bewegen mir Gegenstände aus der Umgebung zu benennen und nachzusprechen. „Das ist ein Opel", sagte ich auf Russisch zu Edeltraud - unsere Unterhaltung fand wie immer vorrangig auf Russisch statt. „Opel, Opel. Das ist ein Opel“, wiederholte sie energisch. „Das ist ein Vogel“, fuhr ich fort. „Ein Vogel“, erklärte ich laut und deutlich. „Vogel. Ein Vogel“, rief Edeltraud.

Plötzlich merkte ich, wie etwas vom Rollstuhl auf den Asphalt fiel. Ich blickte nach unten und musste zu meinem Entsetzen feststellen, dass Edeltraud sich gerade mal eben ihrer Zähne entledigt hatte. Ich hob sie auf und legte sie vorsichtig in meine Tasche, als Edeltraud anfing laut zu schreien, wie sie es oftmals aus heiterem Himmel tat. Die Spaziergänger und Vorbeilaufenden um uns herum warfen sich verwunderte Blicke zu und schienen sich zu fragen, ob denn bei uns beiden alles in Ordnung ist. Habe ich die alte Dame etwa entführt oder ihr weh getan, konnte ich den fragenden und erschrockenen Gesichtern und strengen Blicken der Spaziergänger entnehmen. Ich griff nach dem Rollstuhl und lief unauffällig weiter. Dabei versuchte ich Edeltraud sanft zu beruhigen und gleichzeitig so normal wie möglich auf die Fußgänger um uns herum zu erscheinen. Auch damit sie ruhigen Gewissens weiter gehen konnten (Abb. 1).

Edeltraud beruhigte sich wieder. Ich fuhr fort: „Das ist eine Rose“. Dabei schob ich ihren Rollstuhl ganz nah an die Blume, damit sie daran riechen konnte. „Riecht das nicht unglaublich“, fragte ich sie. Sie schaute mich nur verdutzt an. Ein paar Schritte weiter kam uns eine große Dogge entgegen. Ich wusste nicht, wie Edeltraud auf den Hund reagieren würde, wenn er ihr zu nahe käme. Wenn sie anfing zu schreien, würde das kein gutes Ende nehmen, dachte ich mir. Doch ganz im Gegenteil: Edeltraud schien völlig unbekümmert und fast schon tiefenentspannt, sie hob ganz leicht ihre Hand und deutete eine Streichelbewegung an, 


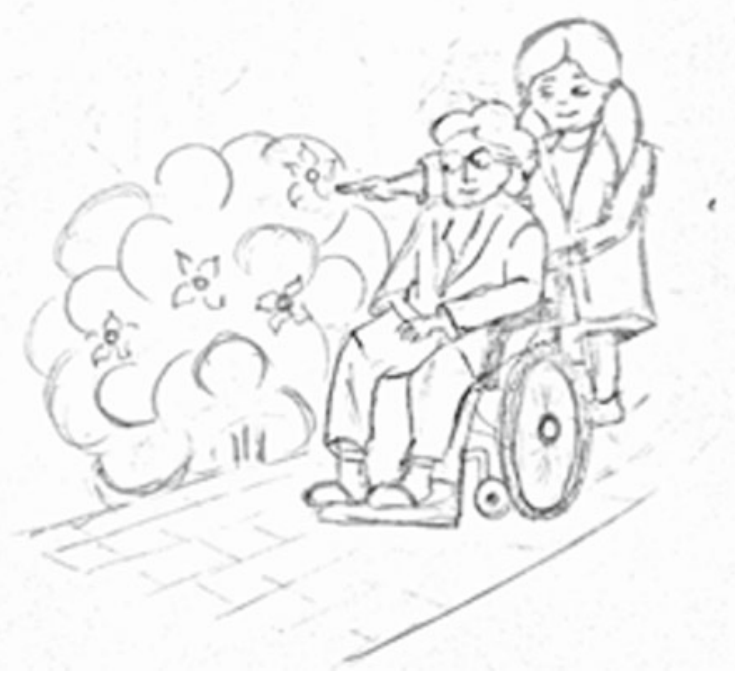

Abb. 1 Ein bewegender Spaziergang. (Bild von Sabina Safarova)

als der Hund langsam an uns vorbeizog und sich Edeltraud näherte, um an ihrer Hand zu schnuppern.

Als wir die Brücke über die Autobahn betraten, blieb ich einen Augenblick stehen, und wir beobachteten die vorbeifahrenden Autos. Aus irgendeinem Grund wirkten die rasend schnell vorbeiziehenden Autos beruhigend auf Edeltraud und mich. Ich erklärte Edeltraud, dass die Autos auf der Autobahn in die Innenstadt fuhren, und im selben Augenblick zeigte sie auf die Autobahn und machte deutlich, dass sie dorthin wollte. Ich erklärte Edeltraud, dass es unmöglich war, mit dem Rollstuhl auf der Autobahn zu fahren, und schmunzelnd kam ich nicht umhin, mir vorzustellen, wie wir zusammen mit dem Rollstuhl so schnell wie die Autos über die Autobahn rasen, während Edeltraud „Schneller, schneller, schneller!“" ruft.

Wir machten uns auf den Rückweg. Ich fragte Edeltraud, ob sie wirklich schon zurück nach Hause wollte. Doch sie hatte keine Lust mehr, weiter spazieren zu gehen und schien erschöpft zu sein. Sie antwortete: „Nach Hause. Kalt!“

Als wir ihr Zimmer nach dem Spaziergang betraten, fanden wir eine ernüchternde Stimmung vor; uns ergriff ein bedrückendes und beklemmendes Gefühl. Das Zimmer erschien trist und verlassen. Ich konnte und wollte noch nicht gehen. 
Die Vorstellung, sie den ganzen Tag in diesen vier Wänden sich selbst zu überlassen, ohne dass uns Beiden noch etwas Freudiges widerfährt, bereitete mir ein beklemmendes und mulmiges Gefühl.

Also setzte ich mich noch etwas zu ihr. Auf ihrem Tisch entdeckte ich einige Bücher, und ich beschloss, Edeltraud etwas daraus vorzulesen. Ich fühlte, dass Edeltraud angespannt und unruhig war. Vielleicht hatte sie ja Redebedarf, und ihr fehlten einfach nur die Worte, um sich auszudrücken, weil sie sie schlichtweg vergessen hatte. Um ihre Stimmung ein wenig aufzuheitern, schnappte ich mir spontan eins von den Büchern, schlug eine beliebige Seite auf und las ihr daraus vor. Es war ein russisches Gedicht von Lermontov, wie ich später herausfinden sollte (Abb. 2).

Als ich anfing, die erste Zeile vorzulesen, wiederholte Edeltraud plötzlich und ohne Aufforderung meine Worte. „Schau Edeltraud, ich lese und du sprichst mir nach“ schlug ich vor. „Das Gedicht ist von Lermontov und heißt ,An Durnov.““ „Wie?“ unterbrach mich Edeltraud. Ich wiederholte: „An Durnov“. „Ich habe genug geliebt, um ewig traurig zu sein. Um glücklich zu sein, habe ich jedoch zu wenig geliebt..." Während ich ihr Zeile für Zeile das Gedicht auf Russisch vorlas, gab sie voller Schwung und Elan und mit all ihrer Energie Zeile für Zeile des Gedichts wieder. Sie hat es mir fast vollständig nachgesprochen bis auf ein

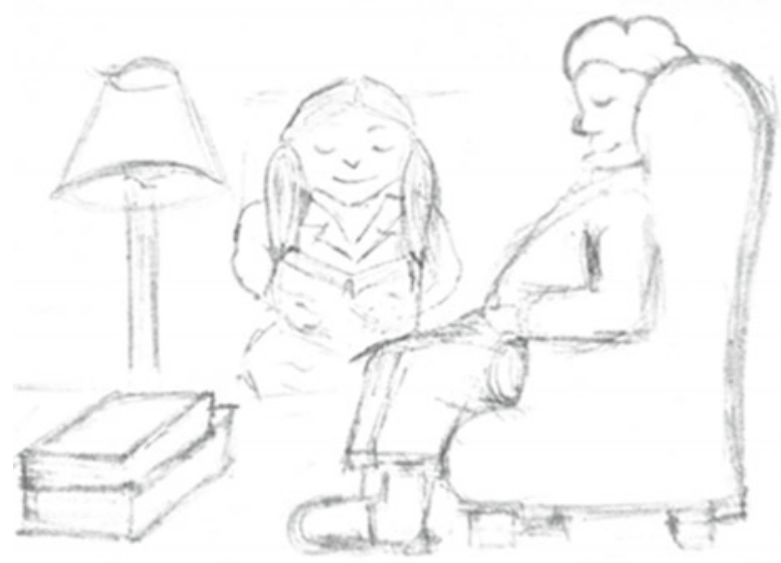

Abb. 2 Ein unvergessliches Gedicht. (Bild von Sabina Safarova) 
paar schwierigere Wörter, bei denen Sie einige Probleme hatte, sie zu verstehen oder auszudrücken.

Ich las weiter vor: „Was bringt es, mir die Seele zu öffnen, denn danach bin ich nicht das, was ich war ..." Edeltraud wiederholte eifrig ,was bringt es, meine Seele zurückzugewinnen, danach bin ich nicht das, was ich war ..."

„Klasse“ lobte ich Edeltraud. „Abends, in der Stunde des ersten Schlafes, als der Nebel funkelte ..." fuhr ich fort. Ich spürte, dass Edeltraud aufblühte, während ich weiterlas. „Worauf?“ fragte sie plötzlich. „Zwischen den Tälern, am Ort. Am Ort" setzte ich fort, ,wo vorher sie gewesen ist. An dem Ort, an dem sie vorher gewesen ist, wandele ich besorgt und allein.“ „Ich gehe besorgt“" wiederholte Edeltraud. Ob sie wohl ihre Angst äußerte, dachte ich mir, als sie die Zeile verändert wiedergab? „Allein“ wollte ich fortfahren, doch Edeltraud schrie plötzlich, dass es ihr genug war: „Es reicht, es reicht“. „Den letzten, den letzten Vers noch bitte" versuchte ich, sie noch einmal zu ermutigen das Gedicht zu Ende zu lesen und begann ,Dann musst du die Augen ...“. „Dann musst du die Augen ...“, sprach Edeltraud in einem etwas traurigeren Ton nach. „Prima“ entgegnete ich ihr liebevoll und las die letzte Zeile vor, während Edeltraud „Es reicht, es reicht“ rief. Voller Freude wiederholte ich nun ,Es reicht, es reicht. Richtig! Wir haben es geschafft." Und legte das Buch schließlich zur Seite.

So ist nicht nur Lermontovs Gedicht unvergessen geblieben, sondern auch dieser unvergessliche und unglaubliche Tag mit Edeltraud.

\section{Das Lachen, das in Erinnerung bleibt - Wie das Lachen von Frau Spiller ein Stück meines Lebens verändert hat}

Milena Ploch

„Wir werden nie wissen, wie viel Gutes ein einfaches Lächeln vollbringen kann.“ - Mutter Theresa

Meine Reise mit dem Projekt UnVergessen und den damit verbundenen Besuchen in Pflegeheimen fing damit an, dass ich am Anfang meines Studiums eine Abwechslung zu meinen theoretischen Seminaren suchte. Als dann das Projekt an mehreren Stellen beworben wurde, war mein Interesse sogleich geweckt. Ich konnte mich anmelden, und schon ging es los.

Zunächst fanden theoretische Sitzungen statt, das heißt, dass der ganze historische Hintergrund und auch das relevante Fachwissen erarbeitet wurden. So durfte ich während dieser Zeit mehr über die Immigration der Polen und Russen 
nach Deutschland erfahren. Außerdem lernte ich wichtige Dinge in Bezug auf die Krankheit Demenz kennen und wie mit dieser umzugehen ist. Ich kann mich noch erinnern, wie mir am Anfang bei dem Gedanken, mit einer an Demenz erkrankten Person zu sprechen, ein bisschen unwohl wurde, da ich nie zuvor so eine Erfahrung gemacht habe. Durch die Vorbereitung konnten mir dann allerdings einige meiner „Ängste“ genommen werden.

Gegen Ende des Wintersemesters fingen die ersten Besuche in den Pflegeeinrichtungen an. Ich kann mich noch gut an meinen ersten Besuch erinnern. Mir wurde von den Mitarbeitern gesagt, dass Frau Spiller ${ }^{4}$ schon ganz aufgeregt sei, mich zu treffen. Sie hätte wohl schon den ganzen Vormittag Polnisch „geübt“, und es würde ihr ein bisschen Sorgen bereiten, da sie doch überhaupt nicht so gut Polnisch sprechen könnte. Dann wurde ich zu ihrem Tisch in der Cafeteria geführt, wo sie mit ihrer Tasse Kaffee saß. Mir fiel sofort ihr herzliches Lachen auf. Ich begrüßte sie zuerst mit „Dzień dobry“ (was „Guten Tag“ auf Polnisch bedeutet) und versuchte, eine Konversation auf Polnisch zu beginnen. Schnell merkte ich aber, dass sie nicht so recht auf Polnisch mit mir reden wollte, und so sprach ich zunächst einmal Deutsch mit ihr. Ich erfuhr, dass ihre Eltern aus Polen kamen und sie so auch etwas Polnisch gelernt hat. Ihr Vater hatte sie sogar auf eine polnische Schule geschickt, wo sie zum Beispiel einige Weihnachtslieder und ein bisschen polnische Grammatik lernen konnte. Nach einiger Zeit war ihr der zusätzliche Unterricht jedoch lästig, und sie hörte auf zur polnischen Schule zu gehen. Besonders deswegen konnte ich mich mit der Geschichte von Frau Spiller identifizieren. Auch ich bin in Deutschland geboren, aber meine beiden Eltern kommen aus Polen und wollten die polnische Kultur und Sprache so gut es ging in unserer Familie erhalten. So wurde auch ich zur polnischen Schule geschickt.

Obwohl Frau Spiller die polnische Sprache als Kind gelernt hat, hatte sie Hemmungen, Polnisch zu sprechen, da sie in der Vergangenheit oft als „Polake“ beschimpft wurde. Sie war aber sehr interessiert daran, wie sich Polen in den letzten Jahrzehnten entwickelt hat und wie ich die dortige politische Lage einschätzen würde. Mich wunderte das, denn ich hatte immer noch dieses Stereotyp im Kopf, dass Senioren sich nur für Bingo, Häkeln und deutsche Schlager interessieren (das mit den deutschen Schlagern war im Falle von Frau Spiller allerdings wahr). So endete mein erster Besuch und ich verabschiedete mich. Sie sagte mir, dass sie sich freuen würde, mich wiederzusehen. Und so kam ich wieder. Und wieder. Die ersten paar Male musste ich mich immer wieder von Neuem vorstellen, aber als ich auf das Polnische zu sprechen kam, wusste sie direkt wieder, wer ich war. Sie war jedes Mal aufs Neue erstaunt darüber, dass die Polen sich auf

\footnotetext{
${ }^{4}$ Der Name wurde geändert.
} 
so eine positive Art und Weise entwickelt haben und in den umliegenden Nachbarländern akzeptiert wurden. Manchmal hatte sie aber ihre Zweifel und fragte mich, ob man denn einfach so laut polnisch sprechen dürfe. Ich vermute, dass der Hass, mit dem sie in der Vergangenheit zu kämpfen hatte, groß gewesen sein musste. $\mathrm{Ab}$ und zu passierte es, dass sie sich an ein polnisches Lied oder Gedicht erinnern konnte. Sie hat es dann immer stolz vorgetragen und war selbst über ihr Gedächtnis erstaunt. Und ich auch.

Außerdem liebte sie das Tanzen, das Lachen und die Musik. Ich war immer wieder beeindruckt, mit was für strahlenden Augen sie von den Abenden erzählte, an denen sie mit ihren Freundinnen zum Tanzen gegangen ist. Sie konnte außerdem in ihrem Alter von 96 Jahren noch Mundharmonika spielen. Ich erinnere mich noch gut daran, wie sie auf der Mundharmonika spielte und ich durch ihr Zimmer getanzt bin. Ihre Freude am Leben und ihr Lachen waren dabei so ansteckend, dass ich mich in ihrer Gegenwart sehr wohl gefühlt habe.

Selbstverständlich gab es auch ein paar Tage, an denen Frau Spiller nicht so gut gelaunt war und Schwierigkeiten hatte, sich zu erinnern. Dazu kamen noch die Schmerzen, die sie in ihren Knochen spürte. An diesen Tagen versuchte ich, so gut ich es nur konnte, sie aufzumuntern. Dies gelang mir manchmal mit einem Lied oder einfach damit, dass ich da war und ihr ein Lächeln schenken konnte.

Mir wurde bewusst, dass wir so viel gemeinsam hatten. Der Unterschied bestand darin, dass ich einige Jahrzehnte später geboren wurde und in einer anderen Welt aufgewachsen bin als Frau Spiller, aber im Grunde gab es zwischen uns trotzdem so viele Berührungspunkte. Wie kommt es dennoch, dass wir so eine Angst oder Vorurteile haben, wenn es um die ältere Generation geht? Warum sprechen wir mit älteren Menschen nicht mehr so über das Weltgeschehen, wie man es mit ihnen getan hat, als sie noch 20 Jahre jünger waren? Natürlich sind Krankheiten wie Demenz Steine auf dem Weg der Verständigung, aber sie hindern uns nicht daran, uns mit einem Menschen hinzusetzen und einfach nur zuzuhören, was er oder sie zu sagen hat. Wir können so viel von unserer älteren Generation lernen. Es wäre schade, wenn wir die Chance verpassen und das Thema Alter in den Hintergrund schieben, bis wir uns selbst irgendwann in dieser Lage befinden. Wenn ich daran zurückdenke, was ich für Berührungsängste hatte, wenn es um Menschen geht, die an Demenz erkrankt sind, weiß ich jetzt, dass diese „Ängste“ aus meinem Unwissen hervorgegangen sind. Als ich Frau Spiller kennengelernt habe und sie meine Freundin wurde, wurde mir klar, dass es so viel mehr Aufklärungsarbeit in Bezug auf die Themen Demenz, Alter und Pflegeheime zu leisten gibt.

So ist mir das Projekt ans Herz gewachsen, und ich werde Frau Spiller, auch wenn sie leider schon von uns gegangen ist, nicht vergessen. Nicht zuallerletzt 
möchte ich mich von ganzem Herzen bei Frau Dr. Karl bedanken, dass sie UnVergessen ins Leben gerufen und eine neue Möglichkeit für diesen generationsübergreifenden Kontakt geschaffen hat. Ich hoffe, dass durch dieses Projekt noch viel mehr Menschen unvergessen bleiben können.

\section{Die Bedeutsamkeit einer Unterhaltung:Wie Dialoge ein Stück Gegenwart und Zukunft im Alter gestalten können}

\section{Lydia Brodovski}

Das vorbeirasende Leben setzt sich aus einem riesigen Potpourri von Erinnerungen zusammen. Doch die Rückblende auf Geschehenes ist weitaus mehr als ein nostalgisches Schwelgen. Das, was wir mit unseren Sinnen wahrgenommen haben, trägt zu unserer Identität bei. Ein Teil unserer Persönlichkeit kann dann verloren gehen, wenn wir die Fähigkeiten des „Erinnerns“ langsam verlieren. Eine individuelle Retrospektive ist aber nicht nur ein Schlüsselfaktor für eine zufriedene Lebensbilanz. Autobiografische Informationen betten sich gleichermaßen in einen größeren Kontext ein und können Antworten auf die verschiedensten sozialen und historischen Fragen geben.

Für viele Senior/innen stellt der Umzug in eine Pflegeinstitution einen großen Einschnitt in ihr Leben dar. Es verändert sich die Wohnsituation. Das soziale Umfeld nimmt neue Formen an: zum einen geht man neue Verbindungen freiwillig ein in Form von neuen Bekannten in der Pflegeeinrichtung, zum anderen findet ein zwangsläufiger Kontakt mit Pflegekräften statt. Die Bewohner/innen teilen sich mit dem Umzug in eine Pflegeinstitution eine Gemeinsamkeit. Ihr Leben erfährt mit dem Einzug einen neuen Umbruch. Der gesamte Tagesablauf trifft auf Veränderungen und mit ihm die menschliche Psyche. Hinter diesen Menschen verbirgt sich selbstverständlich eine Identität mit einer persönlichen Biografie, die durch vergangene, lebensprägende Ereignisse gezeichnet ist. Die gegenwärtigen Bewohner/innen eines Pflegeheimes stellen eine Generation dar, die viele Umbrüche des letzten Jahrhunderts miterlebt hat. Daraus resultiert ein Bild von ihnen, welches sich wie ein Sammelwerk von interessanten Lebenserzählungen von selbst zeichnet. Diese Lebenserzählungen sind nicht nur in ihrer subjektiven Erfahrungswelt pluralistisch. Sie bewahren auch eine Unmenge kulturellen Wissens in sich auf.

Da sich der Umzug in ein Pflegeheim negativ auf die Gedächtnisleistung bei Senior/innen auswirken kann (Specht-Tomann, 2018, S. 184), war es mir in 
meiner Projektteilnahme von besonderem Interesse herauszufinden, welche kulturhistorischen Erinnerungen meine Besuchsperson an die Sowjetunion noch in sich trägt und welchen Einfluss die Immigration nach Deutschland und der Umzug in das Pflegeheim auf sie ausübten, aber vor allen Dingen, inwiefern sich ihr Heimatverständnis verändert hat und welche Umstände dazu beitrugen, dass sich das neue Heim eventuell als ein neues und weiteres Zuhause anfühlt.

Frau Seeberg ${ }^{5}$ ist eine Dame von stolzen 94 Jahren. Geboren in der ehemaligen UdSSR lebte sie den größten Teil ihres Lebens in ihrer Wahlheimat, der Ukraine. Dort arbeitete sie bis zu ihrer Migration nach Deutschland als Ärztin. Frau Seeberg ist eine sehr gesellige Person. Trotz ihres hohen Alters ist sie sehr neugierig, interessiert sich für Musik, Kunst, Literatur und Medizin. Ihre Erzählungen über ihr ehemaliges Leben in der UdSSR zeugen von ihrem guten Erinnerungsvermögen.

Um mit Frau Seeberg ins Gespräch zu kommen und Antworten auf meine Fragen zu erhalten, versuchte ich mich an der Methode der bereits gut erforschten und in der Kranken- und Altenpflege angewandten Methode der Biografiearbeit. Das primäre Ziel der Biografiearbeit ist das Erinnern. Der/die Biograf/in soll im Sammeln und Berichten seiner Erinnerungen individuelle Erlebnisse seiner Lebensbiografie beleuchten und sich somit eigene motivierende Impulse geben. Das Rückblicken auf gemeisterte Krisen, aber auch Höhepunkte in der subjektiven Geschichte bietet dem/der Biograf/in eine wichtige Energieressource. Deshalb dient diese Methode besonders in schwierigen Lebenssituationen als Hilfestellung für den Umgang mit den neuen Umständen (ebd., S. 2). Diese beziehen sich u. a. auf eine Veränderung im sozialen Beziehungsgeflecht. Nachdem die pflegebedürftige Person in ein Pflegeheim eingezogen ist, beschränkt sich der Kreis oft auf die Betreuer/innen, Pflegekräfte, Begleiter/innen und andere Heimbewohner/innen. Der eine oder andere verfällt aufgrund von abnehmenden mentalen und körperlichen Fähigkeiten in einen Identitätskampf und hat mit depressiven Gefühlen und Gedanken, wie „Ich bin unerwünscht“, „Ich bin eingeschränkt“ und „Ich muss sterben“, zu kämpfen (ebd.). Genau da soll die Biografiearbeit einhaken und dem Erzählenden dabei helfen, Veränderungen im Leben zu akzeptieren und eine positive Lebensbilanz zu ziehen. Die Biografiearbeit unterstützt mit ihrer beinah universellen Anwendungsart einen produktiven Prozess, der für die Lebenszufriedenheit von großer Bedeutung ist. Die Zufriedenstellung fügt sich nämlich weiterhin nicht von allein. Sie ,,[...] hängt wesentlich von der Fähigkeit ab, Veränderungen wahrzunehmen und darauf zu reagieren (Anpassung), die eigene Endlichkeit anzunehmen (Rückschau, Lebensbilanz) und die eigene Zukunft in

${ }^{5}$ Der Name wurde geändert. 
gewisser Weise planen und gestalten zu können (z. B. Vorsorgeregelungen treffen, Kontakte pflegen, aktiv bleiben).“ (ebd.). Das Erinnern und Erzählen bietet für den/die Biograf/in also eine Möglichkeit, Geschehenes Revue passieren zu lassen, sich der eigenen Stärken und Schwächen bewusst zu werden, sich mit dem persönlichen Schicksal auszusöhnen und für nächste Generationen einen Zugang zu soziokulturellen Fakten zu eröffnen (ebd., S. 58).

Die Biografiearbeit gehört mittlerweile zum Berufsalltag von Pflegekräften und Begleitpersonen. Nicht nur für den Betreuenden eröffnet sie neue Zugänge, die Pflegekraft profitiert ebenfalls von der Methode. Specht-Tomann (2018, S. 61) unterstreicht dabei die besondere Beziehung zwischen Pflegekraft und Betreuendem, die aufgrund von einem vertraulicheren Verhältnis mithilfe der Biografiearbeit zu einer zufriedeneren Arbeit in der Pflege führt:

„Es macht einen Unterschied, ob Frau R. in Zimmer 5 in erster Linie als „Fall“ mit bestimmten Symptomen und Defiziten wahrgenommen und versorgt wird oder ob durch Einblicke in das Leben dieser Frau emotionale Wärme und Momente der Begegnung möglich werden."

Für Bewohner/innen mit Demenz oder ähnlichen Krankheiten bietet sich das Verschriftlichen von biografischen Daten in verschiedener Form als eine wichtige und interessante Quelle für den Krankheitsverlauf an. Auch alternative Quellen wie Tagebücher oder Chroniken geben Einblick in veränderte Tagesformen. Diese Informationen erfassen wichtige biografische Daten für den/ die Betroffene/n, der/die auf seine Beteiligten den Anschein macht, seine Identität zu verlieren. Neben der identitätsfördernden Funktion für den/die Biograf/in und der beziehungspflegenden Funktion zwischen Bewohner/in und Pflegekraft stellt sich das bereits erwähnte Kriterium der soziokulturellen Fakten als Nebenprodukt der Biografiearbeit heraus.

Eine weitere wissenschaftlich bewährte Methode, historische und soziokulturelle Fakten festzuhalten, ist die Oral History. Hierbei befragen Forscher Personen unter einer gezielten Fragestellung und speichern diese Gespräche in beliebiger Form ab. Im Kontext zur Erinnerungsforschung sind in der Geschichts- und Sozialwissenschaft das Gedächtnis und die Erinnerung seit der zweiten Hälfte des letzten 20. Jahrhunderts zu leitenden Denkfiguren geworden (Csáky, 2004, S. 22). Die mündliche Geschichtsschreibung zielt darauf ab, einzelne Subjekte zu ,historischen Akteuren“ (Tschiggerl, 2019, S. 72) zu machen. Diese Methode entsprang dem Gedanken, die Geschichtsschreibung zu demokratisieren. Die Betrachtung und Einbeziehung des einzelnen Subjektes in die Geschichte erfuhr vor allem 
in den 70er Jahren eine große Aufmerksamkeit. Historikern reichte die Analyse von theoriegeleiteten Gesellschaftsmodellen sowie Aktionen des Staates etc. nicht für die Erklärung von geschichtlichen Prozessen aus. Für die Oral HistoryForscher waren und sind vor allem die Angehörigen von Minderheiten interessant. Autobiografische Texte von Frauen oder Juden waren deshalb von besonderer Wichtigkeit. Neue Ansätze der Oral History verfolgen vornehmlich das Ziel, die Wechselwirkung zwischen Individuen und den gesellschaftlichen Strukturen zu analysieren (Stephan, 2004, S. 2). Bei Anwendung der Methode und der Auswertung ihrer Ergebnisse ist es wichtig zu vermerken, dass objektive historische Fakten nicht das Ziel sind. Eher fokussieren sich die Ergebnisse auf das subjektive Narrativ des/der Erzählenden. Der Wahrheitsgehalt des Erzählten hängt einerseits von der subjektiven Perspektive des/der Erzählenden ab, die z. B. im hohen Alter Gedächtnislücken aufgrund von Traumata oder Demenz enthalten kann. Andererseits beeinflusst der/die Interviewer/in das Gespräch mit seinem Forschungsinteresse und kann es dementsprechend in eine gewünschte Richtung lenken. In dieser Hinsicht unterscheidet sich diese Methode von der Biografiearbeit, die primär auf das Erinnern der Person fokussiert ist. In der Verbindung von der in der Pflege tradierten Biografiearbeit und der Fokussierung auf historische Aspekte wie in der Oral History sah ich eine interessante Herangehensweise und Möglichkeit, dem mich interessierenden Thema der Heimat nachzugehen. Gerade durch die Verquickung von historischen und biografischen Fakten und der Retrospektive im Alter erschien mir dieser Ansatz sinnvoll.

In einer langen Vorstellungsperiode zwischen Frau Seeberg und mir versuchte ich, mir zunächst ein Bild davon zu schaffen, wie weit Frau Seeberg sich gegenüber einer fremden Person öffnen wollte. Schließlich stellten diese Treffen und Gespräche für uns beide etwas komplett Neues dar. Frau Seeberg erzählte gerne und viel, was zur Folge hatte, dass sie zu einigen persönlichen Themen, die sie beschäftigten, sehr ausführlich berichtete. Dennoch hatte sie mit typischen Alterseinschränkungen zu kämpfen. Vor und teilweise während der Treffen litt sie unter enormer Nervosität. Wissend, dass der Mensch im Alter auf viele schwierige Bewusstseinskonflikte trifft (Specht-Tomann, 2018, S. 52), stellte auch für mich die Situation, das Gespräch mit einer motivierenden Haltung zu starten, immer wieder eine kleine Herausforderung dar. Schließlich taute Frau Seeberg im Laufe der Treffen immer wieder auf, freute sich über die Gesellschaft und war jedes Mal ein wenig traurig darüber, wenn das Gespräch sich dem Ende neigte. Aufgrund ihres Redebedarfs möge man meinen, dass Frau Seeberg eventuell wenig Besuch bekommt. Glücklicherweise ist dies nicht der Fall. Sie ist eine von wenigen, die regelmäßig familiäre Gesellschaft genießen darf. Und dennoch ging sie 
in den Gesprächen mit mir auf einige Lebensabschnitte sehr detailliert ein. Einzelne Augenblicke deuteten auf ihre Traurigkeit über Geschehenes hin, die von anderen schönen Erinnerungen aber auch schnell überdeckt wurden.

In den Gesprächen mit Frau Seeberg wurde schnell etwas klar: Es fiel mir schwer, die Gespräche zu lenken und den Redefluss meiner Gesprächspartnerin zu unterbrechen, um sie in die von mir gewünschte thematische Richtung zu lenken. Dies steht im Widerspruch zu der eigentlich angedachten Methode, da diese ohne einen präzise vorbereiteten Fragekatalog, Durchsetzungsvermögen und vor allen Dingen ohne die Übung einer solchen Praxis kaum anzuwenden ist. Diese Diskrepanz stellte sich mir als Herausforderung bei der Teilnahme am Projekt dar. In den mal mehr, mal weniger vertrauten Gesprächen zwischen Frau Seeberg und mir neigte sie dazu, auszuschweifen und emotionale Erinnerungen zu thematisieren. Dies erfuhr ich als Beweis einer bestimmten Form des Vertrauens, öffnete sich Frau Seeberg in solchen Momenten doch mir gegenüber. In meiner Position kam es mir daher nicht in den Sinn, das Gespräch strikt nach meinem Interesse zu lenken. Frau Seeberg fühlte sich wohler, sobald sie über die Themen sprach, die sie sich selbst aussuchte. Dies nahm ich zur Kenntnis und beließ die Gespräche deshalb bei einer Art mündlich tradierten Biografiearbeit. Oft beschränkten sich die Gespräche auf ähnliche Themen. Beide Gesprächsparteien, so schien es mir, waren vollkommen einverstanden und in dem Moment des Gesprächs zufrieden damit. Frau Seeberg hatte eine Möglichkeit, ihre Lebensgeschichten nochmals zu erzählen, eine neue Perspektive einzunehmen und Erfahrungen neu zu überdenken. Dabei erhielt ich die Möglichkeit, eine Komfortzone zu verlassen und mein Ohr auf das Zuhören zu schulen. Aus studentischer Perspektive war es außerdem sehr interessant zu erfahren, welchen Aufwand und welche Mühe die Umsetzung einer bis dahin noch unbekannten und nicht eingeübten Methode - hier der Oral History - kosten kann. Eine weitere Herausforderung muss ebenfalls bedacht sein: In einem rein wissenschaftlichen Verfahren einer Oral History-Studie wird schließlich nicht nur eine Person befragt. Es tritt eine ganze Merkmalsgruppe in den Fokus. Vor diesem Hintergrund bin ich auch im Nachhinein mit der Änderung meines Vorhabens und der Fokussierung auf die Biografiearbeit und das vertraute Gespräch zufrieden. Gerade dadurch wurde ein Austausch ermöglicht, der mir viele neue Perspektiven aufgezeigt hat.

An dieser Stelle möchte ich dieses Projekt würdigen. Es unterstützt mit jedem Gespräch das Erinnerungsvermögen der Bewohner/innen. Nicht jede/r Bewohner/in hat das Glück, von Pflege- und Begleitpersonen umgeben zu sein, die seine/ihre Erinnerungen in der Muttersprache verstehen. Umso mehr erfreuen sich beide Seiten über eine neue Austauschmöglichkeit an wertvollen, subjektiven Memoiren. 


\section{Veränderungen im Gesprächsverhalten eines Menschen mit Demenz über den Zeitraum von drei Jahren}

Yvonne Behrens

\section{Einleitung}

Im Dezember 2016 haben Adam ${ }^{6}$ und ich uns in seinem Zuhause kennen gelernt. Sein Zuhause ist ein Zimmer in einem Bochumer Pflegeheim mit direktem Blick auf eine Kirche und in die Natur. Das Kennenlernen in seiner bekannten Umgebung war ein Vorteil, da wir so viele Gesprächsanlässe finden konnten, die in der Nähe und der Ferne zu sehen waren. So führten wir unser erstes Gespräch über die Kirche, die wir beide gut sehen konnten

Seit unserem Kennenlernen sind nun drei Jahre vergangen. Drei Jahre, in denen wir über uns, unsere Geschichte und so manche sportlichen Ereignisse gesprochen haben. In diesen Jahren haben wir uns nicht nur besser kennen gelernt, nein, ich wurde ebenfalls Zeugin von der Auswirkung einer Demenzerkrankung auf das Gesprächsverhalten von Betroffenen. Diese Eindrücke möchte ich in diesem Artikel beschreiben.

\section{Gespräche im ersten Jahr: Der Gewinn der Muttersprache für die Verarbeitung von Traumata}

Bevor ich Adam das erste Mal traf, wurde ich über seine Geschichte und seine Interessen durch das Pflegepersonal informiert. Adam würde sich besonders für die Themen Automobile und Familie interessieren. Gespräche über den zweiten Weltkrieg, in welchem er nach Deutschland verschleppt wurde, sollte ich vermeiden. Ich nahm mir beides zu Herzen und folgte dem Rat der Pflegekräfte. Ihre Informationen über seine bevorzugten Themen bestätigten sich vollkommen. Besonders das Thema Familie kam immer wieder in unseren Gesprächen vor. Hierbei ging es meist um Wünsche, welche wir noch haben, weniger um die Vergangenheit. Adam schmiedete Pläne und Ziele, die er noch in seinem Leben erreichen wollte, und rührte mich so manches Mal. Die Aussage der Pflegekräfte zur Thematik Krieg erwies sich jedoch als weniger wahr: Es mag sein, dass Adam sich nicht auf Deutsch über den Krieg und seine Erfahrungen unterhalten mochte. Dies traf jedoch nicht auf das Erzählen in polnischer Sprache zu. Bereits bei unserem zweiten, meist polnischsprachigen Treffen sprach er das Thema von sich aus an. Er erzählte viel aus der Kriegszeit, sowohl von schönen als auch traumatischen

${ }^{6}$ Der Name wurde geändert. 
Erlebnissen. Die Geschichten beschrieb er detailliert und in langen Ausführungen. Er verwendete lange Satzstrukturen, unterschiedlichste Wörter und nannte ebenfalls Namen. Letzteres nahm ich zum Anlass, nach dem Wahrheitsgehalt der Geschichten zu recherchieren. Es erstaunte mich, als ich die Personen im Internet wiederentdeckte und somit wusste, dass zumindest ein Teil der Geschichten der Wahrheit entsprach. Adam teilte in dieser Zeit nicht nur viele Erlebnisse, sondern ebenfalls viele Emotionen mit mir. Für mich fühlte es sich so an, als er ob er lange darauf gewartet hatte, diese Erfahrungen mit jemandem teilen zu können, ja, die Last dieser Ereignisse zu teilen. Während ich ihn erzählen ließ und lediglich selten nach weiteren Informationen fragte, um ihn die Tiefe der Gespräche bestimmen zu lassen, baute sich zwischen uns beiden ein Vertrauen auf, welches bis zuletzt bestand.

In dieser Phase sprachen wir meist Polnisch. Es gab jedoch auch immer mal wieder deutschsprachige Tage. Die Gesprächsthemen wurden überwiegend von Adam bestimmt. Er führte die Gespräche und die Richtung, in welche sie gingen. Zudem übernahm er den größten Gesprächsanteil. Unsere Gespräche dauerten zu dieser Zeit bis zu zwei Stunden. Er war sowohl in der deutschen als auch der polnischen Grammatik sicher und zeigte in beiden Sprachen einen reichen Wortschatz.

\section{Gespräche im zweiten Jahr: Sprachliche Sicherheit durch vertraute Themen}

Im Laufe der Zeit haben sich unsere Gespräche verändert. Die Thematik Krieg rückte an den Rand und die Themen Familie und das Alltagsgeschehen rückten in den Fokus nahezu aller Treffen. Die Tiefe der Unterhaltungen wurde weniger abstrakt.

Unsere Gespräche waren häufig mit Objekten aus seinem Zimmer verbunden. Die äußeren Anreize boten uns Themen, welche wir veranschaulichen und somit besser aufgreifen konnten. Wir schauten uns gemeinsam Bücher über Automobile und Polen an und verbanden diese mit unserem Leben. Wo sind wir bereits gewesen, und wo möchten wir gerne einmal hin? Im Gegensatz zum ersten Jahr hat Adam auf diese Fragen nur noch selten eine Antwort. Ein Reiseziel hatte er jedoch noch: Kanada, sein Land der Träume! Dort sollten wir einmal gemeinsam hinreisen! Auch in dieser Phase schmiedete er also noch Zukunftspläne, die wir besprachen. Es gab jedoch auch Tage, an denen Adam wieder die Person aus dem ersten Jahr war, an denen er von früheren positiven Erlebnissen aus seiner Adoleszenz erzählte und wir Bilder von seiner Familie ansahen. An diesen Tagen erinnerte er sich besonders gern an seine Zeit als Bereiter zurück und nannte Namen von Höfen und Pferden, welche er besonders geschätzt hatte. 
Die Sprache unserer Unterhaltungen war immer noch überwiegend Polnisch. Jedoch kam es häufig zu deutschsprachigen Tagen oder zu Sprachmischungen. Wenn Adam auf Deutsch sprach und ich auf Polnisch antwortete, ging er mit der Zeit ebenfalls ins Polnische über. Seine Sätze sind kürzer und weniger inhaltsreich geworden. Er entwickelte jedoch eine Strategie, welche die entstandenen Lücken in seinen Geschichten überdeckten. Er leitete zwar immer noch das Gespräch, befragte mich aber so, dass ich deutlich mehr Gesprächsanteil als im ersten Jahr hatte. Zudem hat unsere Gesprächsdauer abgenommen. Adam ermüdete schneller, sodass wir uns nur noch ca. eine Stunde trafen.

\section{Gespräche im dritten Jahr: Lichtblicke im fortgeschrittenen Rückgang des aktiven Gesprächsverhaltens}

Die Tiefe unserer Gespräche hat stark abgenommen. Im Fokus der Gespräche stand die Thematik Familie, die Adam sehr vertraut und von Beginn an sehr wichtig war.

Anders als zu Beginn behandelten die Gespräche keine Zukunftspläne oder unsere Vergangenheit mehr. Die entwickelte Strategie zur Verdeckung der inhaltlichen Lücken bewahrte sich. Die Gespräche verliefen eher wie ein Frage-AntwortSpiel, welches von Adam geleitet wurde. Größtenteils befragte er mich zu meiner Familie. So erkundigte er sich häufig, ob meine Familienmitglieder zu Besuch seien. Es war ihm stets ein großes Bedürfnis, über die derzeitigen Umstände meiner Familie informiert zu sein. Gespräche über seine Familie führten wir nicht mehr. Umso mehr erfreute es mich, wenn Adam mir eine kurze Geschichte aus seinem Berufsleben erzählte oder aber ein komplexes Wort der deutschen Sprache verwendete. So sagte er im letzten Jahr an einem Tag das deutsche Wort „Zeigefinger“, was nicht nur mich sehr erfreute, sondern ebenfalls alle Pflegekräfte erstaunte. Die Freude, die er uns an diesem Tag mit der Verwendung dieses Wortes machte, zeigt jedoch ebenfalls, wie stark sein aktives Gesprächsverhalten bereits abgebaut war.

Die Sprache unserer Gespräche wechselte zunehmend. Teils unterhielten wir uns auf Deutsch, teils auf Polnisch. Anders als im zweiten Jahr wechselte Adam die Sprache nicht mehr nach einem anderssprachigen Aktivierungsversuch durch mich. Wenn unser Gespräch auf Deutsch begann, führten wir es komplett auf Deutsch. Leider verstummte Adam zunehmend, was nicht bedeutet, dass wir uns nicht mehr austauschen konnten. Je weniger Adam selbst redete, desto mehr kommunizierten wir über unsere Mimik, besonders die Mimik um die Augen herum rückte in den Vordergrund. Auf diese Art brachten wir uns zum Lachen und zeigten uns unsere Verbundenheit. Das über die Jahre aufgebaute Vertrauen begünstigte meiner Meinung nach diese nonverbale Kommunikation. 


\section{Schlusswort: Das Glück der gemeinsamen Zeit}

Das Gesprächsverhalten von Adam hat sich in den letzten drei Jahren stark verändert. Die Person, die mir zu Beginn noch lange und inhaltsreiche Geschichten erzählte, verstummte zuletzt zunehmend. Mit der Zeit haben wir jedoch gelernt, uns ohne Worte zu verstehen. Wir haben Vertrauen aufgebaut und konnten dieses einsetzen und weiter vertiefen.

In den letzten Jahren haben wir viel erlebt: Wir haben uns über unsere Erlebnisse ausgetauscht, Pläne für die Zukunft geschmiedet und viel gelacht. In der letzten Zeit schwiegen wir zwar häufig, doch auch das taten wir gemeinsam. Wir verbrachten gemeinsam Zeit und das ist das, was zählt: Gemeinsam!

Während der Entstehung dieses Artikels ist Adam verstorben. Viele glückliche, spannende oder auch ernste Momente werden mir in wertvoller Erinnerung bleiben. Durch Adam habe ich einen anderen Blick auf das Leben, Vergangenes und sogar die sprachwissenschaftliche Forschung erhalten. Ihm und unseren gemeinsamen Erlebnissen widme ich diesen Artikel.

\section{Auseinandersetzung mit Verlust, Tod und Trauer}

\section{Dorothea Laszczak}

Im Projekt betreute ich zwei Damen, Frau Konrad und Frau Pfeifer ${ }^{7}$. Über ein Jahr lang trafen wir uns regelmäßig, die Gespräche waren teilweise oberflächlich, teilweise gingen sie sehr tief. Jedes Treffen war anders, jedes war besonders.

Tauschen sich zwei Individuen aus, auch über traumatische, glückliche oder wichtige Lebensereignisse, entsteht eine Bindung. Dies kann ich so für mich bestätigen. Der Kontakt war auch daher sehr intensiv, da ich meine MasterThesis über die Biografie von Frau Konrad und Frau Pfeifer verfasste. Ich wählte dafür die Methode der Biografiearbeit und sorgte für möglichst viele entspannte Gesprächsanlässe. Biografiearbeit gibt Einblicke in wichtige Lebensereignisse, in intime Momente, voller Glück, voller Trauer oder Wut. Mitfühlend hörte ich bei vielen Geschichten zu, war Zeuge von Tränen und Schmerz. Das nahm ich mit nach Hause, versuchte diese Erfahrung in meine Arbeit zu integrieren.

Ich erinnere mich noch gut an das letzte Treffen mit Frau Pfeifer. Bei dem Treffen kam mir nicht der Gedanke, dass es das letzte Mal sein könnte, dass wir uns sehen. Ich versprach ihr, dass ich ihr beim nächsten Mal eine verkürzte

\footnotetext{
${ }^{7}$ Die Namen wurden geändert.
} 
Version meiner Master-Arbeit mitbringen würde, damit sie die wichtigsten Punkte herauslesen kann, die bei unseren gemeinsamen Treffen entstanden sind.

Es war leider eine enttäuschende Erfahrung, dass ich von dem Pflegeheim selbst keine Information erhalten habe, dass Frau Pfeifer fortgegangen ist. Diese Information habe ich durch Dritte erfahren müssen, die auch im Altenheim im Rahmen des Projektes tätig waren. Es wäre ein wichtiger Moment für mich gewesen, wenn ich die Möglichkeit bekommen hätte, bei Frau Pfeifers Beerdigung dabei sein zu können, die Möglichkeit, ein letztes Mal Dankeschön für die interessanten und sehr persönlichen Momente auszudrücken und Frau Pfeifer zu verabschieden.

Als ich daraufhin das Pflegeheim um Informationen bat, wo denn Frau Pfeifer beerdigt worden ist, habe ich diese leider nicht erhalten können, sie wussten es wohl selbst nicht. So fing meine Suche nach Frau Pfeifers Grab an. Ich erinnerte mich, wie sie in einer Erzählung erwähnte, dass sie, als sie noch mobil war, oft zur katholischen Messe nach Solingen fuhr. Das war ein erster Ansatz, um nach mehr Informationen zu suchen. Ich suchte alle katholischen Kirchen und Friedhöfe in Solingen zusammen und rief alle nacheinander an.

Der Nachname Pfeifer fiel und eine Dame am Telefon erwähnte, dass vor kurzem eine Dame dieses Namens auf dem Friedhof beerdigt worden ist. Sie gab mir die Adresse durch und die Grabnummer. Ich fuhr am nächsten Tag dorthin. Ich erinnere mich, wie emotional ich an das Thema heranging und voller Erwartungen war, dass ich bei der Suche erfolgreich sein werde. Mir war es wichtig, an Frau Pfeifers Grab eine Kerze anzuzünden und ihr einige Worte von mir zu sagen und für ihren Frieden zu beten, ich wusste genau, wie viele schrecklichen Ereignisse sie im Leben erleben musste - das hatte ich in den vielen Gesprächen mit ihr mehr als einmal erfahren. Als ich auf dem Friedhof ankam - es war ein sonniger und heißer Tag - machte ich mich auf die Suche. Die Enttäuschung war groß, als ich an Feld D, Reihe 6, Nr. 3 stand, das war die Grabnummer, welche mir mitgeteilt worden ist, und nicht den Vor- und Nachnamen von Frau Pfeifer gelesen habe. Ich sah die benachbarten Gräber an und wurde auch nicht fündig. In der nächsten Reihe sah ich ein Grab mit einem unbeschrifteten Grabstein. Vielleicht war dies das Grab von Frau Pfeifer, ich wollte es so gerne glauben. Ich verbrachte über eine Stunde auf dem Friedhof, doch ihr Vor- und Nachname war nicht zu sehen. So zündete ich eine Kerze an einem großen Kreuz mitten auf dem Friedhof an und sagte in Gedanken einige Worte zu Frau Pfeifer und ging.

Gerne möchte ich an dieser Stelle erwähnen, wie wichtig es für das Projekt und die zukünftigen Teilnehmer wäre, dass diese in solchen Situationen wenn irgend möglich informiert werden und so die Möglichkeit zum Abschied bekommen. Es 
können so tiefe und bedeutende Verbindungen im Projekt entstehen, dass es eine schmerzhafte Erfahrung mit sich bringen kann, falls diese Möglichkeit fehlt.

Da ich einige Tage nach der misslungenen Suche nach dem Grab ins Ausland gezogen bin, kam ich leider nicht mehr dazu, mich weiter mit der Suche zu beschäftigen.

Noch bis heute denke ich regelmäßig an Frau Pfeifer und hoffe, dass meine Besuche ihr die Möglichkeit gegeben haben, einige der Ereignisse zu verarbeiten und auf diese Weise loszulassen. Das ist ein wichtiger Gedanke, der dieses Projekt für mich bedeutsam macht.

\title{
Wahrheit oder Lüge? Über den Umgang mit Erzählungen eines Demenzerkrankten
}

\author{
Aldona Rzitki
}

Die Sprache ist das komplexeste Zeichensystem, das es gibt. Trotzdem kommunizieren wir ohne große Mühe erfolgreich miteinander. Die meisten Situationen meistern wir intuitiv. Doch was wäre, wenn man von einer Krankheit betroffen ist, die uns vergessen lässt, wie verschiedene Handlungsabläufe funktionieren? Genau dies verursacht Demenz. Demenziell veränderte Menschen sind je nach Grad der Krankheit eingeschränkt und können in vielen Fällen auch nicht mehr erfolgreich kommunizieren. So können sich Gespräche und damit das Aufbauen einer Beziehung mit betroffenen Personen schwierig gestalten. Das krankheitsbedingte Verhalten der Betroffenen kann man nicht verändern, so liegt es an den Bezugspersonen der Erkrankten, ihr Kommunikationsverhalten zu reflektieren und anzupassen, um positive Beziehungen zu ermöglichen und das Wohlbefinden erkrankter Personen nicht zu gefährden. Mit diesem Kommunikationsverhalten für das Etablieren einer positiven Beziehung setzt sich dieser Beitrag auseinander.

\section{Identität}

Ganz allgemein kann man sagen, dass sich alle Personen voneinander unterscheiden. Der Sozialpsychologe Tom Kitwood zählt einige Dimensionen dieser Unterschiede auf und nennt die Kultur, das Alter, das Geschlecht, die Lebensweise und einige weitere Aspekte. Für ihn ist die persönliche Geschichte des Individuums von Bedeutung: ,Jeder Mensch ist an den inneren Ort, an dem er sich gegenwärtig befindet, auf einem nur ihm eigenen Weg gelangt, und jede Situation an diesem Weg hat dabei ihre Spuren hinterlassen." (Kitwood, 2016, S. 41) 
Für die Interaktion mit Demenzerkrankten ist es von großer Bedeutung, ihre Individualität anzuerkennen und ungeachtet ihrer geistigen Fähigkeit nicht zu vergessen, dass sie eine eigene Geschichte haben, die sie zu dem Individuum gemacht hat, das sie heute sind, auch wenn sie sich durch die fortgeschrittene Demenz selbst nicht mehr an Teile ihrer Vergangenheit erinnern können, die sie geprägt haben. Kitwood betont, dass jeder Mensch unabhängig seiner geistigen Fähigkeiten als eine Person anerkannt werden sollte.

Die jedem Menschen inhärente Rolle ist als Teil der eigenen Identität zu verstehen. Dabei definiert Kitwood diese wie folgt:

„Eine Identität zu haben, bedeutet zu wissen, wer man ist, im Erkennen und im Fühlen. Es bedeutet, ein Gefühl der Kontinuität mit der Vergangenheit und demnach eine ,Geschichte', etwas, das man anderen präsentieren kann, zu haben. Es umfasst außerdem das Schaffen einer Art roten Fadens durch die verschiedenen Rollen und Kontexte des gegenwärtigen Lebens.“ (Kitwood, 2016, S. 148 f.)

Eine Identität zu haben bedeutet also, dass man eine Vergangenheit hat, die man seinem Gesprächspartner präsentieren kann. Dies zählt nach Kitwood zu einem der Grundbedürfnisse, da man dadurch eine Bestätigung seiner selbst erfahren kann (vgl. ebd.). Folglich wird den Interaktant/innen, die sich um das Erfüllen der Grundbedürfnisse bemühen, positiv gegenübergetreten. Dies kann als Grundgerüst für die Konstitution einer positiven Beziehung verstanden werden.

\section{Gesprächsrollen}

Im Laufe seines Lebens sind jedem Menschen diverse Rollen inhärent. Auch viele gesprächsanalytische Ansätze operieren mit diesem aus der Soziologie stammenden Begriff der Rolle. Man kann ihn im Sinne von „Gesprächsrollen“ interpretieren, die flexibel zwischen den Gesprächspartner/innen ausgehandelt werden (Meer \& Pick, 2019, S. 36).

Im Pflegeheim stattfindende Gespräche zwischen demenziell veränderten Menschen und ihren Pfleger/innen unterliegen allerdings anderen interaktionalen Rahmenbedingungen, da Altenheime als eine Institution aufgefasst werden.

„Institutionen sollen hier mit Ehlich \& Rehbein (1977) verstanden werden als gesellschaftliche Apparate, die auf der Grundlage fester Regeln und mithilfe von ausgebildetem Personal je spezifische gesellschaftliche Zwecke erfüllen [...].“ (Becker-Mrotzek, 2009, S. 71)

So gehört auch die Kommunikation der dem Pflegeheim inhärenten Interaktant/innen der Institutionalität an (Becker-Mrotzek, 2009, S. 71). Nach Svenja 
Sachweh sind Pflegeheime vor allem durch ein stabiles Organisationsmuster charakterisierbar (2000, S. 18). Dabei wird jeder Person eine Rolle zugeordnet (Meer \& Pick, 2019, S. 36). In Pflegeheimen gibt es eine starke Hierarchisierung der Interaktant/innen mit einem ausgeprägten Machtgefälle (Sachweh 2000, S. 18). Dies hat zur Folge, dass die Interaktant/innen sich in bestimmten Rollen gegenübertreten, die von einer Asymmetrie geprägt sind (vgl. hierfür die Ausführungen von Karl in Teil I dieses Bandes). Im Pflegeheim würden sich die Gesprächspartner/innen in der Rolle eines Pflegenden und der Rolle der Bewohner/innen wiederfinden. Diese Zuordnung könnte die Handlungsmöglichkeiten der Interaktant/innen vorbestimmen (Becker-Mrotzek, 2009, S. 71), denn mit der Einnahme einer solchen Rolle gehen auch immer Rechte und Pflichten einher. Vor allem für die Kommunikation bedeutet dies, dass sie den Zielen und Zwecken der Institution unterliegt (Sachweh, 2000, S. 75). Diese gibt einen Rahmen vor, in dem sich die Beziehung der Interaktant/innen konstituieren kann (Becker-Mrotzek, 2009, S. 75).

Um Gesprächsrollen aushandeln zu können, müssen mindestens zwei Interaktant/innen in Kontakt treten und miteinander kommunizieren. Dieser Aushandlungsprozess besteht aus zwei Teilen. Denn es wird gezeigt, wie man seine eigene Gesprächsrolle sowie die des Gesprächspartners versteht (Becker-Mrotzek, 2009, S. 75).

\section{Symmetrie}

Nach Sachweh (2003; S. 145 f.) und Posenau (2014, S. 29 f.) können Asymmetrien hinsichtlich des Alters, der physischen und psychischen Fähigkeiten sowie des Wissensstandes, institutioneller Macht, der Mobilität oder der Einschränkung der Bezugsgruppen und schließlich der Abhängigkeit vorliegen (vgl. hierfür Karl „Pflegekommunikation und Morgenpflege“ in Teil I in diesem Buch). Trotzdem gibt es Möglichkeiten, sich einer kommunikativen Symmetrie anzunähern. Nach Sachweh kann man dies mithilfe von verschiedenen Strategien erreichen: Beispielsweise sollte man darauf achten, nicht nur Fragen zu stellen, sondern hin und wieder auch etwas über sich selbst zu erzählen. Vor allem sollte man auch Fragen beantworten, die einem gestellt werden.

Man kann eine positive Beziehungsbotschaft aber auch senden, indem man die Betroffenen um Rat fragt, um Hilfe bittet oder sich nach ihrer Meinung erkundigt. So erleben sie sich nicht nur als Empfänger von Unterstützung, sodass ihr Selbstwertgefühl gestärkt wird (Sachweh, 2019, S. 178).

Wenn sich innerhalb einer Unterhaltung die kognitiven Einschränkungen der von Demenz betroffenen Personen zeigen, kann man die Symmetrie wiederherstellen, indem man eigene Fehler thematisiert. Diese müssen nicht immer 
der Wahrheit entsprechen. So schlägt Sachweh zum Beispiel vor, seine eigenen Fähigkeiten zu relativieren. Diese Strategie wirkt sich nicht nur positiv auf die Erkrankten aus, sondern stabilisiert auch die Beziehung zu ihnen (Sachweh, 2019, S. 178). Asymmetrie kann eine positive Beziehung gefährden. Daher ist es wichtig, sich der verschiedenen Rollen der Interaktant/innen bewusst zu sein und sich um symmetrische Rollen zu bemühen.

\section{Das Image}

Man kann schlussfolgern, dass Identitätsarbeit gleichzeitig ein Teil der Beziehungsarbeit ist, denn diese wird bei der Identitätsarbeit mit jeder Äußerung in der Interaktion vollzogen. Das soziologische Konzept Goffmans (die Image-Theorie) beschreibt diesen Prozess:

„Der Terminus Image kann als der positive soziale Wert definiert werden, den man für sich durch die Verhaltensstrategien erwirbt, von der die anderen annehmen, man verfolge sie in einer bestimmten Interaktion. Image ist ein in Termini sozial anerkannter Eigenschaften umschriebenes Selbstbild - ein Bild, das die anderen übernehmen können.“ (Goffman, 1999, S. 10)

So gibt es auch bei Goffman während der Interaktion die Aufgabe, ein Bild von sich selbst zu vermitteln und sich um das des Anderen zu bemühen. Da Goffman die Aufrechterhaltung des Images nicht als Ziel sieht, sondern als eine Bedingung der Interaktion, führt dies dazu, dass es für die Gesprächsteilnehmer/innen wie auch bei Becker-Mrotzek zwei Orientierungen gibt. Bei Goffman liegt der Schwerpunkt darin, das Image zu wahren. Er spricht von einer defensiven Orientierung, bei der die Interaktant/innen ihr eigenes Image wahren, und von einer protektiven, bei der es darum geht, das Gesicht des anderen zu wahren (Goffman 1999, S. 19). Dies ist für die Kommunikation mit Demenzerkrankten eine wichtige Erkenntnis, da der angemessene Umgang mit betroffenen Personen beinhaltet, ihre kognitiven Probleme während der Kommunikation abzufedern. Dafür hat Sachweh Strategien erarbeitet, die genau dieses von Goffman beschriebene protektive Verhalten umsetzen.

Für Sachweh kann die Interaktion mit Pflegebedürftigen gesichtsbedrohend oder gesichtsschonend realisiert werden. Dabei stützt sie sich auf Theorien von Brown und Levinson (1987), die die Face-threatening acts (FTAs) einführen (Posenau, 2014, S. 43). Darunter verstehen sie: 
„Kommunikative Bezugnahmen [...], die entweder das „,positive face“ (das Selbstbild des Interaktanten) bedrohen oder das ,negative face“ (seinen Anspruch auf Selbstbestimmung des eigenen Handelns, auf Ungestörtheit und auf die Unverletztheit des eigenen Territoriums) (ebd., 61) in negativer Weise tangieren." (Posenau, 2014, S. 43)

Gesichtsschonende Strategien versuchen diese negativen kommunikativen Bezugnahmen abzuschwächen oder zu vermeiden. Dies ist wichtig, um die kommunikative Asymmetrie bei der Kommunikation auszugleichen und so eine positive Beziehung zu konstituieren.

\section{Protektive Strategien am Beispiel der Konfabulation}

In den vorherigen Kapiteln wurde bereits ausgeführt, dass das Erzählen über die eigene Identität ein Grundbedürfnis ist. Von Demenz betroffene Personen haben spätestens im mittelschweren Stadium stark ausgeprägte kognitive Störungen, wodurch auch das Altgedächtnis anfängt zu verblassen. So fallen einige der ihnen vorher inhärenten Rollen weg. Sie vergessen je nach Krankheitsgrad Teile ihrer eigenen Geschichte. Wenn man durch solch eine kognitive Einschränkung mehr Erinnerungen abzurufen versucht, als tatsächlich noch vorhanden sind, können sogenannte Konfabulationen entstehen. Darunter können erdachte Aussagen verstanden werden, wie sie auch von meiner Bezugsperson im Projekt UnVergessen produziert werden. Dies illustriert ein Auszug aus meinem Erfahrungsbericht, den ich im Rahmen meiner Projektteilnahme verfasst habe:

Immer wieder bemerkte ich, dass Herr Schimanski ${ }^{8}$ mir Dinge über sich selbst erzählte, die nicht stimmen. Hierbei gibt es zwei Arten von falschen Aussagen. Die einen sind flüchtig und werden nicht erneut erwähnt. Zumeist entstehen sie aus der Situation heraus. Als wir bei einem Treffen beispielweise über Politik sprachen, erwähnte Herr Schimanski, dass Angela Merkel mit ihm verwandt sei. Andere Aussagen hingegen tauchen in Gesprächen immer wieder auf. Zum Beispiel erwähnte Herr Schimanski oft selbstständig die Themen Fußball und Heimat und erzählte dabei, dass Oliver Kahn sein Cousin sei, anschließend berichtete er, dass Kahn in seinem Dorf in seiner Straße gewohnt habe und führte seine Geschichte weiter aus. Die erfundenen Details stimmten bei jedem Treffen überein.

Meine Erklärung für ein solches Gesprächsverhalten ist, dass Herr Schimanski durch das Verblassen seiner Erinnerungen einen Teil seiner Identität verloren hat, sodass er seinem Gegenüber immer weniger zu präsentieren hat. Da er aber etwas zu erzählen haben möchte, überlegt er sich neue Geschichten. Da Identität in diesem Sinne nicht nur das eigene Verständnis über einen selbst, sondern bis zu

\footnotetext{
${ }^{8}$ Der Name wurde geändert.
} 
einem gewissen Grad auch etwas ist, das einem von anderen verliehen wird, sorgt Herr Schimanski dafür, ein alternatives Selbstbild zu erzeugen, damit eine neue Identität entstehen kann. Er versucht, sein Selbstbild mit diesen Konfabulationen auszugestalten, um Anerkennung von seinem Gegenüber zu bekommen.

Man könnte nun auf viele Arten auf diese erdachten Geschichten reagieren. Man könnte die Konfabulation als Lüge darstellen und Herrn Schimanski damit konfrontieren. Dies wäre aber eine gesichtsbedrohende Strategie. Um also an dieser Stelle richtig zu reagieren, muss man sich das Ziel der Unterhaltung vor Augen führen. Es geht nicht darum, Fakten abzustecken. Ich kommuniziere mit Herrn Schimanski, um sein Wohlbefinden zu verbessern und eine positive Beziehung zu konstituieren. Dies erreiche ich eher, wenn ich die Bedürfnisse des Anderen erfülle. In diesem Fall ist es das Bedürfnis nach Anerkennung. Ich entscheide mich also für die gesichtsschonende Strategie, indem ich vorgebe, ihm zu glauben. An dieser Stelle muss erwähnt werden, dass dies im Grunde eine Lüge ist.

Deshalb möchte ich diesen auf den ersten Blick unethisch anmutenden Aspekt meines Handelns beleuchten. Sachweh benutzt die Terminologie der "Notlüge“. Nach Wahrig Deutsches Wörterbuch wird dieser Terminus definiert als „eine Lüge aus Höflichkeit, um den anderen nicht zu kränken" (Sachweh, 2019, S. 366). Demnach kann das Mitspielen bei einer Konfabulation als gesichtsschonende Strategie angesehen werden, die das Bedürfnis nach Anerkennung befriedigt. Für Sachweh gehört zu einem angemessenen Umgang mit demenzerkrankten Personen, ,ihre subjektive Realität und ihr Selbstbild zu akzeptieren und zu bestätigen [...]“" (Sachweh, 2019, S. 175).

Außerdem gibt es noch einen weiteren Grund für eine solche Reaktion. Man kann nicht wissen, ob Herr Schimanski sich seiner Falschaussagen bewusst ist. Aufgrund seiner kognitiven Einschränkungen könnte es sein, dass er seine Konfabulation selbst glaubt. Sachweh schreibt dazu:

\begin{abstract}
„Streng genommen bedeutet das also, dass man einem [...] dementiell [...] Erkrankten [...] ganz in seine Welt folgt und versucht, ihm dort zu begegnen, ohne ihn wieder in unsere Realität zerren zu wollen. Eine Notlüge zu verwenden heißt letztlich, die jetzt und hier geäußerten Erfahrungen, Gefühle, Bedürfnisse und Denkweisen von Menschen mit Demenz ernst zu nehmen und respekt- und würdevolle Wege zu beschreiten, um emotional angemessen auf sie zu reagieren. Es bedeutet nötigenfalls auch, die eigenen Vorstellungen von ,richtig“ und „falsch“ je nach Situation zurückzustellen.“ (Sachweh, 2019, S. 367)
\end{abstract}

Auf die Erzählungen des Anderen einzugehen kann also durchaus seine Berechtigung haben. Sachweh ist aber der Meinung, dass Notlügen nur dann angewendet werden sollten, wenn sie zum Besten des Erkrankten sind (Sachweh, 2019, S. 
375). Man sollte nicht nur lügen, um es sich einfacher zu machen. Es ist also immer der Kontext der Lüge entscheidend (Sachweh, 2019, S. 380). Außerdem gibt Sachweh zu bedenken, dass man die Lüge authentisch vermitteln muss. Wenn dies nämlich nicht der Fall ist und die Lüge als solche erkannt wird, könnte das einen konträren Effekt erzielen, indem es für Misstrauen sorgt und der Beziehung schadet (Sachweh, 2019, S. 382). Es soll nicht unerwähnt bleiben, dass es auch Gegner von Lügen in der Interaktion mit Demenzerkrankten gibt. Allerdings bin ich der Ansicht, dass Notlügen in dieser speziellen Kommunikationssituation zu befürworten sind, wenn sie das Gesicht meines Gegenübers wahren und so für sein Wohlbefinden sorgen.

\section{Mein kleines Fazit}

Immer wieder denke ich an Herrn Schimanski und unsere Gespräche zurück. Er erzählte mir viele (im wahrsten Sinne des Wortes) unglaubliche Geschichten und wir führten einige lustige Unterhaltungen. Ich empfand die Treffen als wirkliche Bereicherung.

Das Wort ,Bereicherung' mag zunächst widersprüchlich klingen, da man sich fragen könnte, warum ausgedachte Erzählungen bereichern sollen. Ich habe aber mittlerweile verstanden, dass der Mehrwert sich dadurch ergibt, dass wir eine schöne gemeinsame Zeit hatten. Ob die Inhalte der Geschichten nun stimmten oder ausgedacht waren, ist letztendlich für unsere Beziehung irrelevant. Viel wichtiger ist es, sich darauf einzulassen und seinem/seiner Gesprächspartner/in die Wertschätzung entgegenzubringen, die er/sie verdient.

\section{Literatur}

Becker-Mrotzek, M. (2009). Mündliche Kommunikationskompetenz. In M. Becker-Mrotzek (Hrsg.), Mündliche Kommunikation und Gesprächsdidaktik (Bd. 3, S. 66-83). SchneiderVerl. Hohengehren. https://doi.org/10.1515/9783110231724.

Brown, P., \& Levinson, S. (1987). Politeness. Some universals in language usage. Cambridge University Press.

Csáky, M. (2004). Die Mehrdeutigkeit von Gedächtnis und Erinnerung: Ein kritischer Beitrag zur historischen Gedächtnisforschung. In Digitales Handbuch zur Geschichte und Kultur Russlands und Osteuropas (Bd. 9, S. 1-30). https://epub.ub.uni-muenchen.de/603/ 1/csaky-gedaechtnis.pdf.

Gagarina, N., Klassert, A., \& Topaj, N. (2010). Sprachstandstest Russisch für mehrsprachige Kinder. ZAS Papers in Linguistics, 54, 4-55.

Goffman, E. (1999). Interaktionsrituale. Über Verhalten in direkter Kommunikation (5. Aufl.). Suhrkamp. 
Kitwood, T. (2016). Demenz: Der person-zentrierte Ansatz im Umgang mit verwirrten Menschen (7. Aufl.). Hogrefe.

Meer, D., \& Pick, I. (2019). Einführung in die Angewandte Linguistik: Gespräche, Texte, Medienformate analysieren. Springer-Verlag.

Posenau, A. (2014). Analyse der Kommunikation zwischen dementen Bewohnern und dem Pflegepersonal während der Morgenpflege im Altenheim. Verlag für Gesprächsforschung.

Sachweh, S. (2000). "Schätzle, hinsitze!”: Kommunikation in der Altenpflege. Lang.

Sachweh, S. (2003). ,so frau adams $\downarrow$ guck mal $\downarrow$ ein feines bac-spray $\downarrow$ gut $\uparrow$ : Charakteristische Merkmale der Kommunikation zwischen Pflegepersonal und BewohnerInnen in der Altenpflege. In R. Fiehler, \& C. Thimm (Hrsg.), Sprache und Kommunikation im Alter (S. 143-160). Verlag für Gesprächsforschung.

Sachweh, S. (2019). Spurenlesen im Sprachdschungel: Kommunikation und Verständigung mit demenzkranken Menschen. Hogrefe AG.

Specht-Tomann, M. (2018). Biografiearbeit in der Gesundheits-, Kranken- und Altenpflege. Springer. https://doi.org/10.1007/978-3-662-54393-1.

Stephan, A. (2004). Erinnertes Leben: Autobiographien, Memoiren und Oral-HistoryInterviews als historische Quellen. In Digitales Handbuch zur Geschichte und Kultur Russlands und Osteuropas (Bd. 7, S. 1-30). https://epub.ub.uni-muenchen.de/627/1/Ste phan-Selbstzeugnisse.pdf.

Tschiggerl, M., Walach, T., \& Zahlmann, S. (2019). Geschichtstheorie. Springer. https://doi. org/10.1007/978-3-658-22882-8.

Open Access Dieses Kapitel wird unter der Creative Commons Namensnennung 4.0 International Lizenz (http://creativecommons.org/licenses/by/4.0/deed.de) veröffentlicht, welche die Nutzung, Vervielfältigung, Bearbeitung, Verbreitung und Wiedergabe in jeglichem Medium und Format erlaubt, sofern Sie den/die ursprünglichen Autor(en) und die Quelle ordnungsgemäß nennen, einen Link zur Creative Commons Lizenz beifügen und angeben, ob Änderungen vorgenommen wurden.

Die in diesem Kapitel enthaltenen Bilder und sonstiges Drittmaterial unterliegen ebenfalls der genannten Creative Commons Lizenz, sofern sich aus der Abbildungslegende nichts anderes ergibt. Sofern das betreffende Material nicht unter der genannten Creative Commons Lizenz steht und die betreffende Handlung nicht nach gesetzlichen Vorschriften erlaubt ist, ist für die oben aufgeführten Weiterverwendungen des Materials die Einwilligung des jeweiligen Rechteinhabers einzuholen.

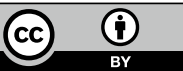

